Check for updates

Cite this: RSC Adv., 2018, 8, 34634

\title{
Nanocharacterization of liposomes for the encapsulation of water soluble compounds from Cordyceps sinensis CS1197 by a supercritical gas anti-solvent technique $\dagger$
}

\begin{abstract}
G. M. Shashidhar*a and B. Manohar (iD b
Nano-liposomes were designed for the sustained release of water soluble compounds from C. sinensis CS1197 using a supercritical gas anti-solvent (SC-GAS) method at various pressures, temperatures and Tween 80 concentrations. The SC-GAS method was compared to the Bangham method of liposome production in terms of mean diameter, coefficient of uniformity $\left(C_{\mathrm{u}}\right)$, encapsulation efficiency, morphology, viscosity and actual energy required for liposome formation. Liposome production via the SC-GAS method under optimized formulation conditions, i.e., 180 bar; $50{ }^{\circ} \mathrm{C}$; $0.75 \%$ Tween 80 ; and a depressurization rate of 25 bar $\mathrm{min}^{-1}$, yielded nano-liposomes exhibiting the lowest $C_{u}$ value $(1.10 \pm 0.012)$ with a mean diameter of $0.072 \pm 0.002 \mu \mathrm{m}$ and better encapsulation efficiencies of $75.48 \pm 2.5,74.9 \pm 2.1$ and $70.23 \pm$ $2.9 \%$ for adenosine, cordycepin and polysaccharides, respectively. Nano-liposomes were characterized using FTIR, XRD, DSC and TGA techniques. The stability indices and viscosities of the prepared liposome suspensions indicated good stability of up to 2 months and near-Newtonian behavior. The in vitro release of CS1197 water soluble compounds exhibited biphasic and sustained release patterns.
\end{abstract}

\author{
Received 12th September 2018 \\ Accepted 24th September 2018 \\ DOI: 10.1039/c8ra07601d \\ rsc.li/rsc-advances
}

\section{Introduction}

Cordyceps sinensis (CS) is a highly valued traditional Chinese medicine with various health benefits. $C$. sinensis is an excellent nourishment option for the kidneys, lungs and liver and is an effective aphrodisiac. Water soluble compounds, such as nucleosides and polysaccharides, from $C$. sinensis possess unbelievable pharmacological properties. ${ }^{1,2} C$. sinensis water extract has been effectively used to treat various pathological conditions and has also been used in various functional food preparations. C. sinensis based therapies may face difficulties, such as a low therapeutic effect, due to their polar nature, which may cause increased intracellular absorption. Nucleosides in C. sinensis water extract are small molecules $(<500 \mathrm{D})$, which are affected by rapid clearance and suboptimal biodistribution. To address current challenging tasks, like the development of sustained and targeted drugs or active principles, the development of nano-carriers with the best information from nano-science is a sound potential approach and is a major focus of current research.

Among the available nano-carriers, nano-liposomes have attracted much attention as potential carriers of drugs. ${ }^{3}$ The idea

${ }^{a}$ Sarda Gums \& Chemicals, Pali-Marwar - 306401, India. E-mail: shashigalaxy@ gmail.com; Fax: +912932 281324

${ }^{b}$ Department of Food Engineering, CSIR-CFTRI, Mysore, India

$\dagger$ Electronic supplementary information (ESI) available. See DOI: 10.1039/c8ra07601d of nano-processing $C$. sinensis based active ingredients for the development of smart drug delivery systems, like those involving liposomes, has become an emerging trend to achieve targeted and sustained release. This is because liposomes are potent drug delivery systems because of their ability to meet several requirements, such as the suitable stability of active molecules, yield and efficiency of drug encapsulation, reproducibility of the micro/ nano-particle quality and the drug release profile, and residual levels of organic solvent in the particles. Basically, liposomes are composed of one or more bilayers of amphipathic molecules (e.g., phospholipids) surrounded by an aqueous continuous phase. The major advantage of liposomal encapsulation is that both hydrophilic and hydrophobic molecules can be intercalated. ${ }^{4}$ Moreover the surfaces of liposomes offer flexibility for any modifications to achieve the site specific unloading of active principles and/or drugs.

Many methods have been developed for liposome production. ${ }^{5}$ The supercritical method is a novel approach through which liposomes can be made in a single step by making use of supercritical carbon dioxide $\left(\mathrm{SCCO}_{2}\right){ }^{6}$ Zarena et al. (2011) reviewed variants of the method to produce submicron- and nano-particles using $\mathrm{SCCO}_{2}$, such as: the rapid expansion of supercritical solutions (RESS); the gas anti-solvent (GAS) method; the precipitation with compressed anti-solvent process (PCA); solution enhanced dispersion via supercritical fluids (SEDS); and the particles from gas-saturated solutions (PGSS) method. ${ }^{7}$ Further, GAS is a more universal process and offers 
greater flexibility than the other variants because the compounds to be encapsulated do not need to be dissolved in $\mathrm{SCCO}_{2}$. Both hydrophilic and hydrophobic compounds can be entrapped in lipid vesicles. Kadimi et al. (2007) claimed an intercalation efficiency (IE) of $20 \%$ for amphotericin B and, in a similar study, an IE of $28 \%$ for seabuckthorn leaf extract with a narrow range of size distribution (0.48-1.07 $\mu \mathrm{m})$, using a $\mathrm{SCCO}_{2}$ method. ${ }^{8,9}$ Liposome preparation in batch mode via a modified supercritical fluid method improved particle sizes in the range of 265 and $214 \mathrm{~nm} .^{\mathbf{1 0 , 1 1}}$ Further efforts have been made to decrease the particle size to $146 \mathrm{~nm}$, with the superior characteristics of liposomes over traditional methods, and a similar study proposed the production of liposomes in a continuous mode using a supercritical method. ${ }^{12,13}$

Preliminary attempts to design liposomes using the GAS method $^{8,9}$ have been successful in the submicron range $(<1 \mu \mathrm{m})$ in the author's lab. To the best of our knowledge, this is the first study reporting the fed-batch mode development of nano-sized liposomes for the entrapment of water soluble compounds from C. sinensis CS1197. The major objective of the current study is to optimize the formulation variables, i.e., pressure, temperature, and Tween 80 concentration, to design nano-liposomes via a SC GAS method. The nano-liposomes produced via the SC method were well characterized using FTIR, DSC, TGA and XRD techniques. Liposomes prepared with the SC method were compared to those obtained with the Bangham method.

\section{Experimental}

\section{Materials and methods}

Materials. The CS1197 strain of $C$. sinensis was obtained from NTCC, FRI, Dehradun, India. Water extract (WE) from dried CS1197 mycelium was obtained following a method reported earlier. ${ }^{14}$ Soy lecithin (SL) was obtained from $\mathrm{M} \mathrm{s}^{-1}$ Shakti soyas Ltd., TN, India, and comprised a natural mixture of phospholipids (phosphatidylcholine (PC), phosphatidylethanolamine (PE), phosphatidylinositol (PI) and phosphatidic acid (PA)), glycolipids and triglycerides. Cholesterol (Chol, 99\% purity) and Tween 80 were purchased from Sigma Chemical (Bangalore, India). Other reagents of analytical grade were from Rankem (Bangalore, India) and Merck (Bangalore, India). Food grade $\mathrm{CO}_{2}$ (purity of 99.9\%) was from local suppliers (Mysore, India).

Preparation of nano-sized liposomes via a SC-GAS method. The apparatus for and process of liposome production for the encapsulation of water soluble compounds from $C$. sinensis CS1197 (CS liposomes) based on GAS principles are detailed elsewhere. ${ }^{5}$ Briefly, the process involves two major steps: (a) the $\mathrm{SCCO}_{2}$ assisted drying of the liposomal contents; and (b) the hydration of lipids under pressure to form large unilamellar vesicles (LUVs) following nano-sized liposome production through depressurization. C. sinensis CS1197 WE was mixed with liposomal contents such as SL and Chol $(3: 1 \mathrm{w} / \mathrm{w})$ in the extract : liposomal content ratio of $1: 10(\mathrm{w} / \mathrm{w})$. The liposomal contents were dissolved using a solvent mixture of chloroform and ethanol $(3: 1 \mathrm{v} / \mathrm{v})$ at a ratio of $1: 3(\mathrm{w} / \mathrm{v})$ and loaded into a $500 \mathrm{ml}$ high pressure reactor vessel (Berghof Autoclave, HPR, Germany). Initially, experiments were carried out in batch mode to optimize a favorable operating range for pressure, temperature and surfactant content. The experimental process was optimized via tuning the process parameters, such as pressure, temperature and Tween 80 concentration, between 50 and 200 bar, 40 and $65{ }^{\circ} \mathrm{C}$, and 0 and $3 \%$, respectively.

Preparation of CS liposomes via a thin-film method. Liposomes were also prepared via a conventional thin film process, known as the Bangham method. ${ }^{15}$ The liposomal contents were dissolved in a mixture of chloroform and ethanol $(3: 1, \mathrm{v} / \mathrm{v})$ at a ratio of $1: 20(\mathrm{w} / \mathrm{v})$. The organic solvents were removed under reduced pressure in a rotary evaporator at $65^{\circ} \mathrm{C}$ (above the lipidtransition temperature). After $1 \mathrm{~h}$ of equilibration, a phosphate buffer solution (PBS) (pH 7.2)/Tween 80 (0.75\%, w/v) solution was introduced under vacuum and hydrated for $2 \mathrm{~h}$ for better encapsulation. ${ }^{9}$

\section{Characterization of CS liposomes}

Mean particle diameter and coefficient of uniformity $\left(C_{\mathbf{u}}\right)$. The mean particle size was determined using a particle size analyzer (model: S3500, Microtrac Inc., USA). Each measurement was carried out in triplicate and the results are expressed as mean $\pm \mathrm{SD}$, where $\mathrm{SD}$ represents the mean error. $C_{\mathrm{u}}$ is used as a criterion in the particle size distribution of soil to provide the index of gradation. It is known that the higher the value of $C_{\mathrm{u}}$, the larger the range of particle size and uniformity indicated, when $C_{\mathrm{u}}$ is between 1 and $3 .{ }^{16}$ In the present study, $C_{\mathrm{u}}$ is used as a criterion to define the disparity of liposome particles, where a value of less than 4 is defined as being uniformly distributed and above 4 as showing a broad distribution range. $C_{\mathrm{u}}$ is defined as the ratio of $D_{60}$ to $D_{10}$. The values for $D_{60}$ and $D_{10}$ were obtained from particle size distribution data.

Determination of the encapsulation efficiency (EE). A known amount of CS liposome suspension appropriately diluted was used to determine the EE. Liposomes of adenosine, cordycepin and polysaccharides were measured for their EEs. Adenosine and cordycepin content were measured via HPLC and the polysaccharide content was measured via a phenol sulfuric method detailed elsewhere. ${ }^{\mathbf{1 4}}$ The diluted solution was subjected to centrifugation at $7500 \mathrm{rpm}$ for $1 \mathrm{~h}$ at $4{ }^{\circ} \mathrm{C}$. A free extract aliquot was then separated for analysis. The encapsulation efficiency was calculated using the equation:

$$
\text { Encapsulation efficiency }(\%)=\frac{C_{1}-C_{2}}{C_{1}} \times 100 \%
$$

where $C_{1}$ is the initial content (adenosine, cordycepin and polysaccharides) in the $C$. sinensis CS1197 water extract used for encapsulation and $C_{2}$ is the amount of free content present in the liposome suspension.

\section{Morphology of CS liposomes}

Phase contrast microscopy. The liposome samples were diluted with triple distilled water in order to obtain better images. About $3 \mu \mathrm{l}$ of diluted liposome was placed on a glass slide with a micro-syringe and then covered with a cover slip. The slide was then subjected to phase contrast microscopy (Olympus BX-5, Japan) including a digital camera to capture 
images. All liposome samples were observed under $100 \times$ magnification.

Scanning electron microscopy (SEM). Scanning electron microscopy (model: LEO 435 VP; LEO Electron Microscopy Ltd., Cambridge, UK) was used to observe the topography and surface morphology of the liposomes. Samples were freeze dried first on SEM stubs and analyzed for liposome particle sizes and shapes.

Liposome stability index (LSI). The emulsifying activity of the surfactant and the interaction of the lipid mixture under supercritical $\mathrm{CO}_{2}$ for tailoring the nano-liposome particles were assessed via determining the LSI using a turbidimetric method. Freshly made liposomes were diluted appropriately with triple distilled water just before measuring the absorbance at $500 \mathrm{~nm}$ using a UV-visible spectrophotometer (UV-1800, Shimadzu, Japan) with $1 \mathrm{~cm}$ path length cuvettes. The liposomes were stored in an incubator for $24 \mathrm{~h}$ at a temperature of $37{ }^{\circ} \mathrm{C}$, followed by measuring the absorbance at a wavelength of $500 \mathrm{~nm} .{ }^{17}$ The turbidity and LSI of the liposomes were calculated using equations, as mentioned in an earlier report. ${ }^{5}$

Rheological examination. The viscosities of the CS liposomes were determined using a controlled stress rheometer (Rheostress 6000, Haake, Karlsruhe, Germany) at $25 \pm 0.1{ }^{\circ} \mathrm{C}$, using a coaxial cylinder attachment (spindle no. $\mathrm{Z} 41$ ) by applying the shear rate in a linear manner from 0.1 up to $100 \mathrm{~s}^{-1}$ over a span of $60 \mathrm{~s}$. All measurements were carried out in duplicate and the apparent viscosity of the liposomes was calculated at a shear rate of $100 \mathrm{~s}^{-1} .{ }^{18}$

Differential scanning calorimetric (DSC) analysis. DSC (PerkinElmer DSC 8000, Norwalk, CT, USA) was used to determine the phase transition behavior, such as the onset temperature $\left(T_{\mathrm{o}}\right)$, melting temperature $\left(T_{\mathrm{m}}\right)$, completion temperature $\left(T_{\mathrm{c}}\right)$, and enthalpy $\left(\Delta H, \mathrm{~J} \mathrm{~g}^{-1}\right)$, of free $C$. sinensis, CS1197 WE, and empty and C. sinensis CS 1197 WE encapsulated nanoliposomes. A $5 \mathrm{mg}$ sample was placed in an aluminum pan and DSC analysis was carried out at a nitrogen flow rate of 15 $\mathrm{ml} \mathrm{min}{ }^{-1}$ and a heating rate of $10{ }^{\circ} \mathrm{C} \min ^{-1}$ from 30 to $300{ }^{\circ} \mathrm{C}$. An empty aluminum pan was placed on the reference platform.

Thermogravimetric analysis (TGA). TGA of samples was carried out using TGA Q50 apparatus (TA instruments, New Castle, DE USA). The experiments were performed at a heating rate of $10{ }^{\circ} \mathrm{C} \mathrm{min}{ }^{-1}$ under a nitrogen flow of $150 \mathrm{ml} \mathrm{min}{ }^{-1}$. Dried C. sinensis CS1197 water extract and encapsulated nanoliposomes were weighed (5-10 mg) accurately in a hanging aluminum pan and the percentage weight losses of the samples were monitored from 30 to $600{ }^{\circ} \mathrm{C}$.

$\mathrm{X}$-ray diffraction (XRD) analysis. X-ray diffraction analysis of C. sinensis CS1197 WE and encapsulated CS liposomes was carried out to observe the purity and crystalline structures using a Reguka Miniflex diffractometer (1.5426 ̊) with $\mathrm{Cu} \mathrm{K} \alpha$ radiation, operating at $30 \mathrm{kV} / 15 \mathrm{~mA}$. All measurements were performed at room temperature within the $2 \theta$ range of $0-80^{\circ}$ and at a speed of $1^{\circ} \mathrm{min}^{-1}$.

Fourier transform infrared (FT-IR) analysis. The interactions between C. sinensis CS1197 water soluble compounds and the lipid membranes were evaluated via Fourier transform infrared spectroscopy (FT-IR). FT-IR spectra were recorded with $\mathrm{KBr}$ pellets on a FTIR-8400S spectrometer (Shimadzu, iR solution 1.30, Shimadzu Corporation, Japan) between 4000 and $400 \mathrm{~cm}^{-1}$.

Storage stability studies. CS nano-liposomes prepared via both the SC-GAS and Bangham methods were kept for observation for 2 months at different temperatures $\left(4,25\right.$, and $\left.37^{\circ} \mathrm{C}\right)$ and $\mathrm{pH}$ values $(2,4,7$, and 10). The samples kept at different temperatures and $\mathrm{pH}$ values were withdrawn and checked for stability at regular intervals via measuring the EE, size, viscosity and $C_{\mathrm{u}}$ of the CS nano-liposomes. Fresh samples were considered as controls.

Oxidative stability of nano-CS liposomes. Determinations of oxidative stability were based on the peroxide value (PV) as per the AOCS official method Cd 8-53. A liposome sample ( $0.1 \mathrm{~g})$ was mixed with a solvent mixture of chloroform : methanol $(7: 3 \mathrm{v} /$ $\mathrm{v})$, followed by the addition of $50 \mu \mathrm{l}$ of both $10 \mathrm{mM}$ xylenol orange solution and ferric chloride solution. After incubation for $5 \mathrm{~min}$ in the dark, the absorbance of the mixture was taken at $560 \mathrm{~nm}$ using a UV/vis spectrophotometer (UV-1800, Shimadzu, Japan). The standard curve is plotted from a series of Fe(III) standard solutions. PVs were expressed as milliequivalents (meq) $\mathrm{O}_{2} / \mathrm{kg}$ liposome: ${ }^{19}$

$$
\mathrm{PV}=\frac{\left(A_{\mathrm{S}}-A_{\mathrm{B}}\right) \times m_{\mathrm{i}}}{W \times 55.84 \times 2}
$$

where $A_{\mathrm{S}}$ is the absorbance of the sample, $A_{\mathrm{B}}$ is the absorbance of the blank, $m_{\mathrm{i}}$ is the inverse of the slope of the standard curve, and $W$ is the weight of the sample (g).

In vitro release. To estimate the adenosine, cordycepin and polysaccharides release under in vitro conditions, the leakage of these compounds from the liposomal interior was monitored via a membrane dialysis method. A dialysis bag was filled with $3 \mathrm{ml}$ of CS liposome suspension, immersed in $100 \mathrm{ml}$ of PBS (pH 6.8), and incubated in a water bath at $37^{\circ} \mathrm{C}$ with stirring at $100 \mathrm{rpm}$. At intervals of $0.25,0.5,1,2,4,6,8,10,12,24,36$ and $48 \mathrm{~h}, 2 \mathrm{ml}$ of the surrounding buffer was sampled and, at the same time, an equal volume of PBS was replenished. The concentration of adenosine, cordycepin and polysaccharides in the extract was determined as described above. The dialysis behavior of free $C$. sinensis CS1197 WE mixed with PBS was examined using the same method. The rate and release mechanism of C. sinensis CS1197 water soluble compounds from the prepared nano-liposomes was analyzed through fitting the in vitro release data using the following kinetic models:

$$
\begin{aligned}
& \text { (1) } \ln C=\ln C_{0}-K_{1} t \text { (first order model) } \\
& \text { (2) } Q=K_{2} t^{1 / 2} \text { (Higuchi model) }
\end{aligned}
$$

where $C$ is the amount of drug released at time $t ; K_{1}$ is the first order release rate constant $(\% / \mathrm{h})$ and $K_{2}$ is the diffusion rate constant.

Statistical analysis. Statistical analysis of the data was conducted using Microsoft ${ }^{\circledR}$ Excel 2010. Data were reported as mean \pm standard deviation (SD). Analysis of variance (ANOVA) was conducted and significant differences between means were obtained via Tukey's multiple comparison tests at a significance level of $p<0.05$. 


\section{Results and discussion}

\section{Preparation of nano-sized CS liposomes via the SC-GAS method}

The phenomena involved in liposome formation using the $\mathrm{SCCO}_{2}$-gas anti-solvent technique have been illustrated earlier. ${ }^{5}$ The basic principle of the GAS method involves the saturation of lipid components with $\mathrm{SCCO}_{2}$, reducing solubility in organic solvents and resulting in precipitation. The lipid drying step was optimized at $15 \mathrm{~min}$, during which lipid saturation with $\mathrm{SCCO}_{2}$ facilitates the rapid diffusion of $\mathrm{CO}_{2}$, with the attainment of an expanded lipid bilayer with the complete removal of remnant solvent. Hydration under pressure and equilibration for $1 \mathrm{~h}$ yields LUVs with a bimodal particle size distribution. The depressurization of the SC liquid to reduce the large unilamellar vesicles (LUVs) to small unilamellar vesicles (SUVs) on the nanoscale is an essential step in the GAS technique. A slow depres-

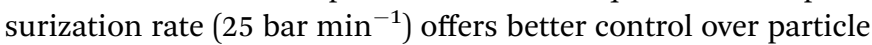
size and distribution, as observed in a previous study. ${ }^{5}$ Similar observations were made by other authors. ${ }^{\mathbf{2 0}, 21}$ The importance of the depressurization of the SC liposome suspension near the lipid transition temperature is discussed later.

\section{Effects of pressure}

The effects of pressure at a constant temperature $\left(50{ }^{\circ} \mathrm{C}\right)$ and Tween 80 concentration $(0.75 \%)$ on the mean diameter, EE, and $C_{\mathrm{u}}$ of liposomes prepared via the SC-GAS technique are shown in Fig. 1A. The pressure was varied between 50 and 200 bar and it was found that increasing the pressure has a significant $(p<$ 0.05) positive effect on particle size, EE and $C_{\mathrm{u}}$. The extent of free and diffused $\mathrm{CO}_{2}$ in the phospholipid bi-layer is the determining parameter for the particle size of liposomes. Fig. 1A indicates that a lower pressure of $<80$ bar is not enough to reduce the particle size to the nano-level $(<100 \mathrm{~nm})$ due to the ineffective diffusion of $\mathrm{CO}_{2}$ in the lipid bi-layer. Liposome formation at pressures of 50 and 80 bar cannot ensure the sphericity of particles due to the unequal diffusion of $\mathrm{CO}_{2}$ throughout the lipid layer and its ineffectiveness in the complete unpacking of the bi-layer structure to discrete lipid molecules. Low pressure treatment yields liposomes with a broad range particle size distribution (Fig. 2A). As the pressure was increased from 120 to 200 bar, there was a significant reduction in particle size and this pressure range is effective for producing spherical particles possessing a narrow particle size distribution range (Fig. 2B). Lisha Zhao and co-workers reported operating pressure range to obtain spherically shaped submicron liposomes. ${ }^{\mathbf{1 0 , 1 1}}$ A higher pressure range facilitated the $\mathrm{CO}_{2}$ /aqueous/Tween80/phospholipids quaternary system to become more favorable for uniform dispersion of phospholipid molecules, allowing them to unite together after depressurization to form a larger number of nano-sized liposomes per unit volume; thus a better EE can be achieved. Increasing the pressure from 50 to 200 bar drastically reduces the liposome size from $0.978 \pm 0.003$ to $0.099 \pm 0.004 \mu \mathrm{m}$, with a greater number of liposomes per unit volume achieving a maximum EE of 75.48 $\pm 2.45,74.78 \pm 3.2$ and $70.31 \pm 2.9$ for adenosine, cordycepin and polysaccharides, respectively.

The current configuration of the nano-liposome production setup was proven to engineer unimodal nano-liposomes over the tested pressure range (50-200 bar), provided the pressure inside the pressure vessel was necessarily kept constant during depressurization. Throughout depressurization, $\mathrm{CO}_{2}$ was released uniformly from every portion of the liquid phase,

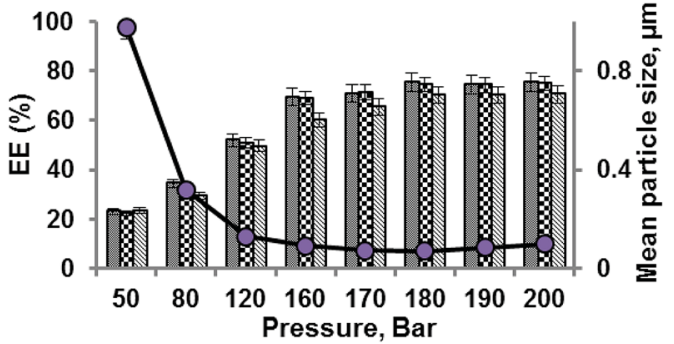

(A)

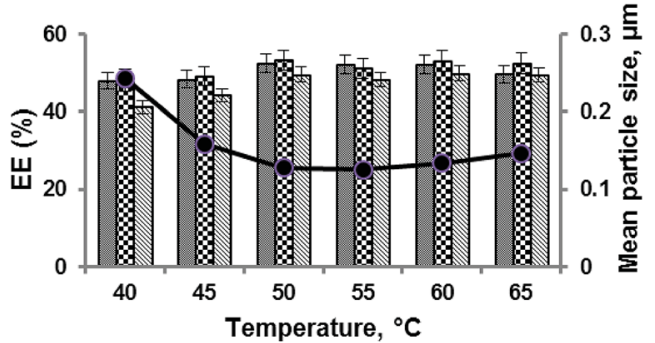

(B)

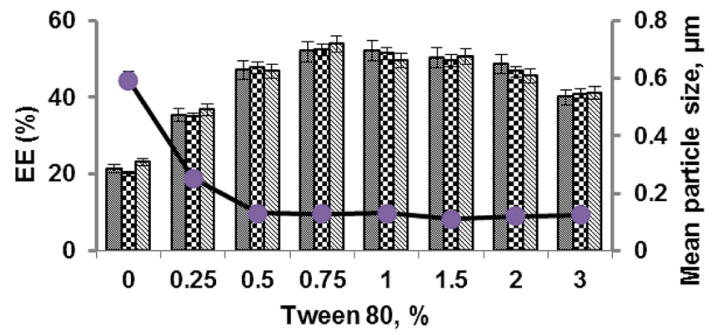

(C)

Fig. 1 Effects of pressure (A), temperature (B) and Tween $80 \%$ concentration (C) on the mean particle size and the liposomal encapsulation efficiency of adenosine, cordycepin, and polysaccharides in CS1197 WE. and $\longrightarrow$ : mean diameter. 


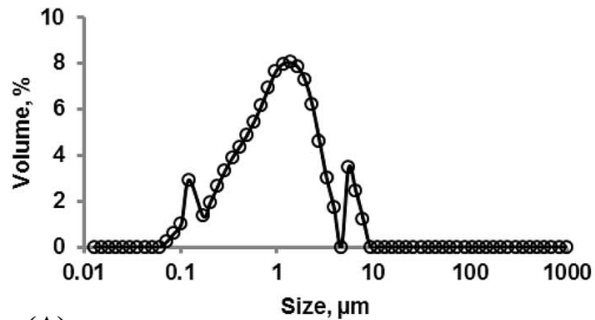

(A)
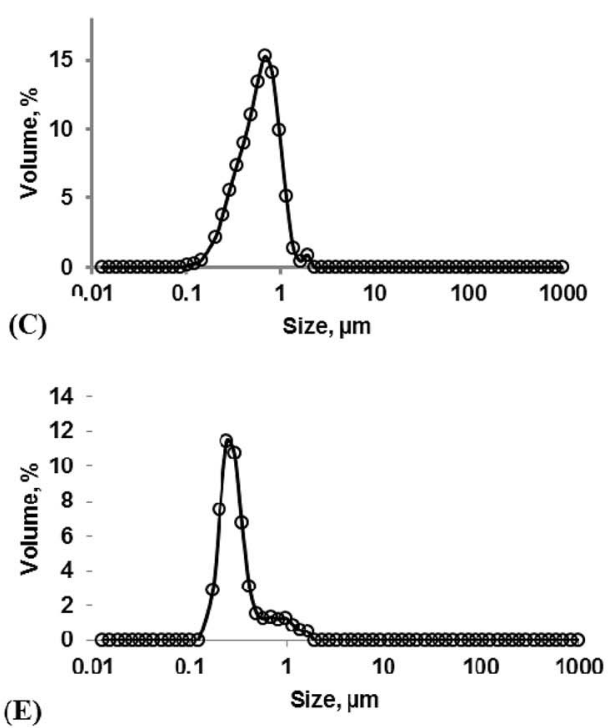

$(\mathbf{E})$

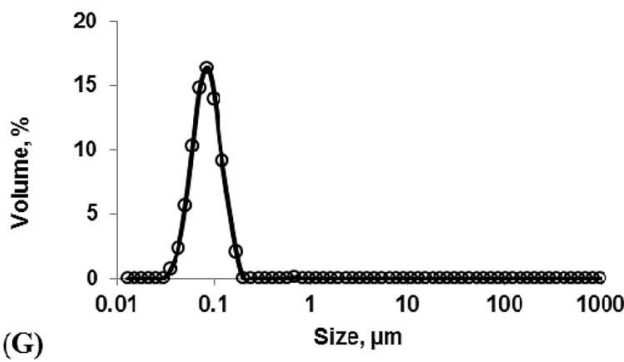

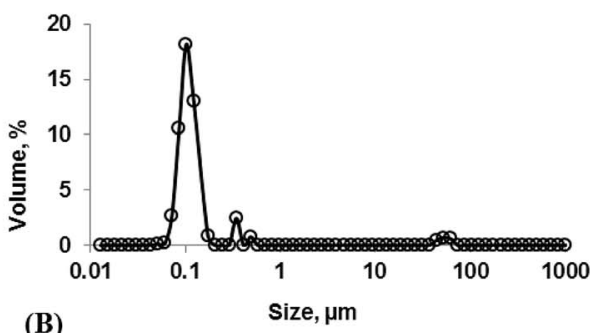
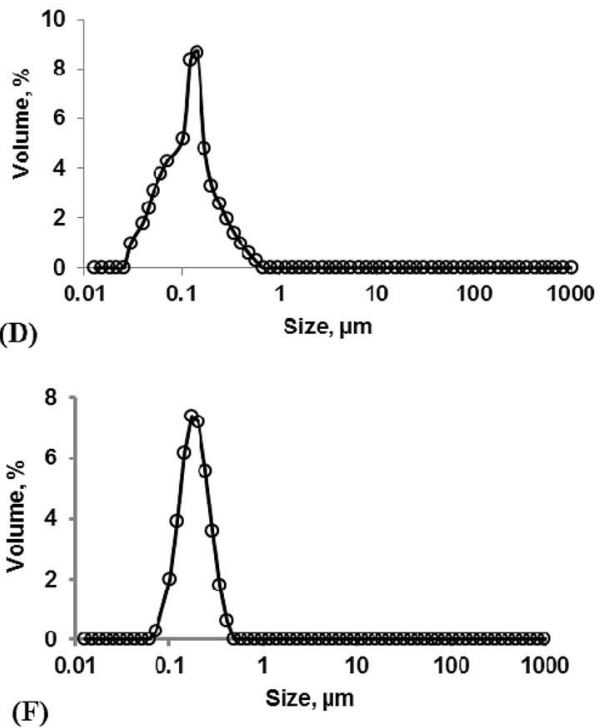

(F)

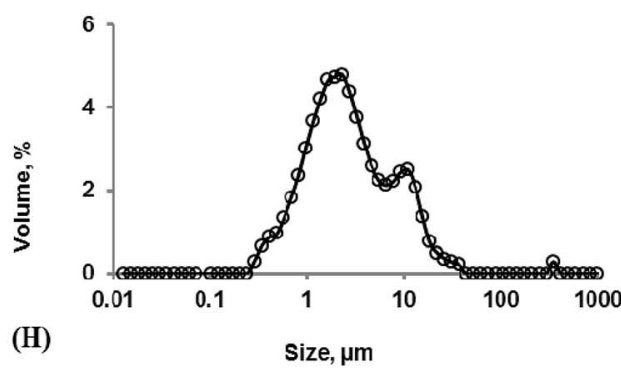

Fig. 2 The size distribution of liposomes obtained: at pressures of 50 bar (A) and 200 bar (B); at Tween 80 concentrations of $0 \%$ (C) and $4 \%$ (D); at temperatures of $40{ }^{\circ} \mathrm{C}(\mathrm{E})$ and $65^{\circ} \mathrm{C}(\mathrm{F})$; under optimized conditions of 180 bar, $50{ }^{\circ} \mathrm{C}$ and $0.75 \%$ Tween $80(\mathrm{G})$; and via the Bangham method (H).

leading to each particle being influenced by dense $\mathrm{CO}_{2}$ and leading to a low $C_{\mathrm{u}}$. The increasing pressure reasonably improved the uniformity of the liposomes from $8.32 \pm 0.013$ to $1.19 \pm 0.012$ (Table $\mathrm{S} 1 \dagger$ ) over the tested pressure range. Further experiments were carried out at pressures between 160 and 200 bar to select the best effective pressure for nano-liposome production. The liposomes produced at a pressure of $180 \mathrm{bar}$ were the smallest liposomes, with a mean particle size of 0.072 $\pm 0.003 \mu \mathrm{m}$ (Fig. $2 \mathrm{G}$ ) and $C_{\mathrm{u}}$ of $1.10 \pm 0.012$ (Table S1†), with better EE.

\section{Effects of Tween 80 concentration}

The presence of a surfactant (Tween 80 ) plays an important role in the $\mathrm{CO}_{2}$ /aqueous/Tween 80/phospholipids quaternary system, wherein the concentration of Tween 80 majorly governs the extent of lipid dispersion into the aqueous phase, which helps liposome recovery during the depressurization step. The concentration of Tween 80 at a constant pressure (120 bar) and temperature $\left(50{ }^{\circ} \mathrm{C}\right)$ was varied between 0 and $3 \%$ to evaluate its effect on the mean particle size, EE and $C_{\mathrm{u}}$ of liposomes (Fig. 1C). Even though one can obtain liposomes in the absence of a surfactant, the particle mean diameter is larger $(0.593 \pm$ $0.005 \mu \mathrm{m}$ ) with a broad particle size distribution (Fig. 2C) and poor EEs of $21.34 \pm 3.1,20.12 \pm 1.9$ and $23.2 \pm 2.7$ are achieved for adenosine, cordycepin and polysaccharides, respectively. This is due to the fact that no repulsive force exists around the liposomes after depressurization to stop coalescence, and moreover there is incomplete dispersion of lipids in the aqueous phase affecting the $\mathrm{CO}_{2}$ /aqueous/surfactant/lipids quaternary homogenous phase, and about $50 \%$ (by wt) of the liposome material still remained in the pressure vessel, thus affecting the EE. Experiments were carried with small amounts of Tween $80(0.25 \%)$ to produce liposomes, where the particle size was reduced to $0.252 \pm 0.003 \mu \mathrm{m}$ and $C_{\mathrm{u}}$ was $4.65 \pm 0.012$ 
(Table S1†). Increasing the concentration of Tween 80 from 0.25 to $0.75 \%$ shows a positive effect, from which a minimum particle size of $0.129 \pm 0.002 \mu \mathrm{m}$ (Fig. 1B), maximum EEs of $51.96 \pm 1.98,52.36 \pm 2.6$ and $53.78 \pm 2.1$ for adenosine, cordycepin and polysaccharides, respectively, and improved $C_{\mathrm{u}}$ of $3.14 \pm 0.011$ (Table S1†) were obtained for $0.75 \%$. Liposome production using a Tween 80 concentration in the range of 0.25 to $2 \%$ resulted in an 80 to $90 \%$ (data not shown) liposome yield, and the rest remained in the vessel due to foam formation, which was unavoidable. A further increase in surfactant concentration from 1 to $3 \%$ resulted in a slight increase in the mean diameter (Fig. 2D). This may be due to continued foam formation causing negative effects. ${ }^{22}$

\section{Effects of temperature}

Variations in temperature at a constant pressure (120 bar) and Tween 80 concentration (1\%) around the phase transition temperature $\left(40-65{ }^{\circ} \mathrm{C}\right)$ involve melting transitions of lecithin, which is due to van der Waals interactions between adjacent lipid molecules. In the absence of $\mathrm{CO}_{2}$, lecithin exists in a lamellar gel phase at lower temperatures, whereas at higher temperatures it changes to a fluid liquid-crystalline phase. In the presence of dense $\mathrm{CO}_{2}$ and cholesterol, the gel-fluid phase transition temperature is reduced and the fluid liquidcrystalline phase region is broadened. ${ }^{23}$ In a high pressure system, whatever the temperature, the fluid liquid-crystalline phase is favoured, which is required for SC mediated liposome production as it enables liposomal encapsulation even at mild temperatures. ${ }^{24}$ In the present study, the effects of temperature at a constant pressure and surfactant content were evaluated with regards to the particle size, EE and $C_{\mathrm{u}}$ of liposomes (Fig. 1B). Temperature has a significant effect $(p<0.05)$ on the $\mathrm{CO}_{2}$ /aqueous/Tween 80/lipids quaternary system, as an increase in temperature from 40 to $50{ }^{\circ} \mathrm{C}$ results in the reduction of the particle size from $0.243 \pm 0.005$ to $0.128 \pm 0.002 \mu \mathrm{m}$ (Fig. 2E). This is due to an increased density of $\mathrm{CO}_{2}$ under the interactive effects of pressure (120 bar) and temperature (45 and $50{ }^{\circ} \mathrm{C}$ ), which creates the most favorable conditions for increased $\mathrm{CO}_{2}$ solubility in the aqueous phase and the formation of small particles. But at higher temperatures $\left(55-65{ }^{\circ} \mathrm{C}\right)$ the particle size was increased to $0.147 \pm 0.004 \mu \mathrm{m}$ (Fig. $2 \mathrm{~F}$ ) and there was a slight decrease in the EE; this is due to the fact that elevated temperatures decrease the cohesive energy density of $\mathrm{CO}_{2}$, lowering its solubility in the aqueous phase and also causing the partitioning of the surfactant towards the aqueous phase, creating a slightly higher $\mathrm{CO}_{2}$-lecithin phase and a lower surfactant-aqueous phase. ${ }^{25}$ The unequal availability of surfactant results in the aggregation of liposomes during depressurization. An increase in temperature also results in the formation of a greater number of fine particles, thus increasing the EEs to $52.40 \pm 1.68,53.25 \pm 2.9$ and $49.57 \pm 2.6$ for adenosine, cordycepin and polysaccharides, respectively. $C_{\mathrm{u}}$ decreased from $3.18 \pm 0.012$ to $2.80 \pm 0.015$ as the temperature increased from 40 to $50{ }^{\circ} \mathrm{C}$ (Table S1 $\dagger$ ) and later increased with temperature to $2.98 \pm 0.018$, due to phase separation causing a non-uniform distribution of surfactant molecules around the liposomes.
Hence, an optimal temperature of $50{ }^{\circ} \mathrm{C}$ is recommended for the production of nano-sized liposomes, where better size uniformity and EE can be obtained.

\section{Importance of depressurization and depressurization at the phase inversion temperature to the particle size and EE}

The importance of the depressurization step lies in the conversion of LUVs to SUVs via creating a pressure drop through micrometric high pressure tubing. Experiments were carried out under optimized conditions, i.e., $180 \mathrm{bar}, 50{ }^{\circ} \mathrm{C}$ and $0.75 \%$ Tween 80 , to understand the importance of depressurization on the particle size and EE. Liposome samples were collected from the pressure vessel just after the hydration step, and liposomes exhibited a large multimodal size distribution with a $C_{\mathrm{u}}$ value of $3.01 \pm 0.021$ with EEs of $59.23 \pm 2.3,57.13 \pm$ 2.1 and $53.12 \pm 1.9$ for adenosine, cordycepin and polysaccharides, respectively. A unique feature of the current configuration of the SC-GAS process is that particle formation and encapsulation happen twice, i.e. during hydration under pressure and after depressurization, which is one possible way to obtain liposomes with superior characteristics exhibiting better EE.

It is well understood that the presence of dense $\mathrm{CO}_{2}$ in the $\mathrm{CO}_{2} /$ water/surfactant/lipids quaternary system facilitates the phase transition of lecithin at a mild temperature. During the depressurization step, the release of $\mathrm{CO}_{2}$ causes cooling of the liquid phase, creating a sudden shock, which is un-favorable for the re-assembling of lipid molecules to form liposomes. Due to an increase in the phase transition temperature, phospholipids undergo a phase transition from a fluid liquid-crystalline phase to a lamellar gel phase. Fig. 3M clearly proves that depressurization of the SC liquid phase at ambient temperature causes the formation of fewer liposomes, and most of the lipid molecules remain discrete, exhibiting poor EEs of $47.34 \pm 2.5,45.98$ \pm 3.1 and $41.56 \pm 2.1$ for adenosine, cordycepin and polysaccharides, respectively. So it is recommended that depressurization to atmospheric pressure occurs at a certain temperature above the lipid phase transition to obtain the highest number of small uniform liposomes.

\section{Characterization of CS nano-liposomes}

Morphologies of the liposomes. Liposomes prepared via Bangham and SC-GAS methods were observed under a phase contrast microscope to characterize the morphologies and shapes of the liposomes. All samples were observed under $100 \times$ magnification to confirm the occurrence of nano-sized and spherical liposomes, as per the data obtained using the particle size analyzer. From Fig. 3A-E it can be seen that increasing the pressure at a constant temperature, Tween 80 concentration

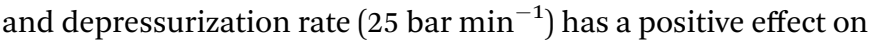
particle size reduction and the sphericity of the liposomes. Fig. 3F-J also suggests that increasing the concentration of Tween 80 from 0 to $2 \%$ improved the particle dispersion in the aqueous phase, establishing a stabilized liposome system and inhibiting particle growth. The occurrence of large 


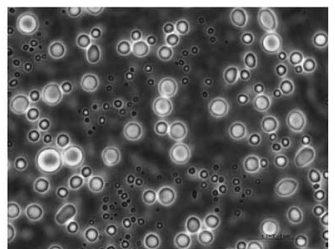

(A)

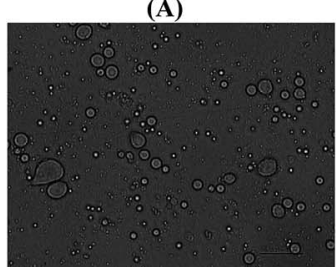

(F)

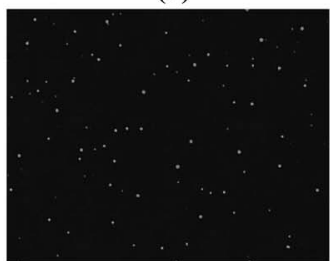

(K)

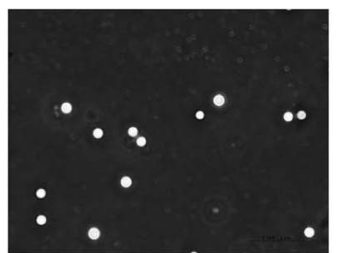

(B)

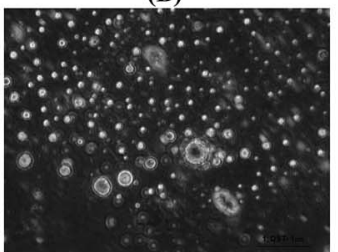

(G)

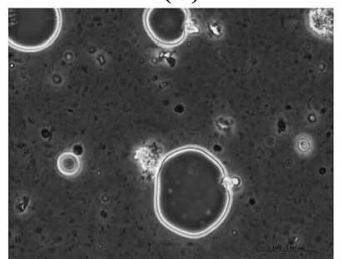

(L)

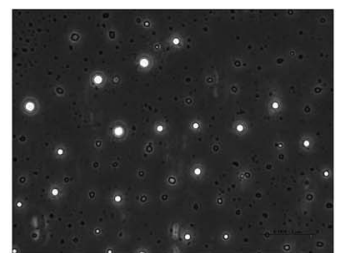

(C)

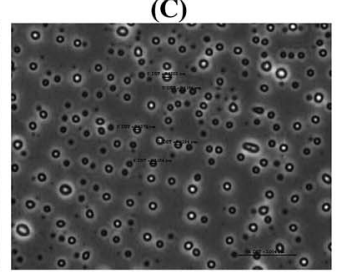

(H)

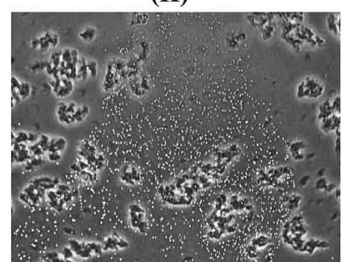

(M)

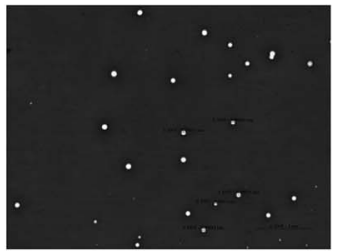

(D)

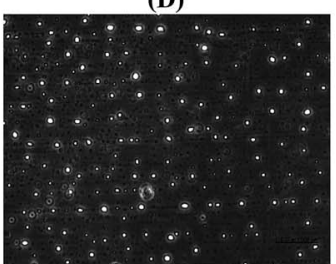

(I)

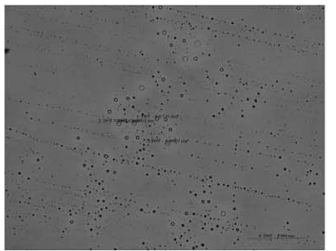

(E)

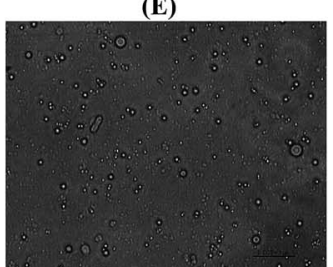

(J)

Fig. 3 Phase contrast micrographs of CS1197 WE liposomes prepared using different formulation variables: (A-E) pressures ranging from 50200 bar, $50{ }^{\circ} \mathrm{C}, 0.75 \%$ Tween 80; (F-J) Tween 80 concentrations of $0,0.25,0.5,1$, and $2 \%, 170$ bar, $50{ }^{\circ} \mathrm{C} ;(\mathrm{K}) 180$ bar, $50{ }^{\circ} \mathrm{C}, 0.75 \% \mathrm{Tween} 80$; ( $\mathrm{L}$ ) using the Bangham method; and (M) at 180 bar, $50{ }^{\circ} \mathrm{C}, 0.75 \%$ Tween 80 , with depressurization at room temperature. All liposome samples were observed under $100 \times$ magnification.

multilamellar liposomes with irregular shape from the Bangham method was evident in Fig. 3L.

The occurrence of very much smaller sized liposomes $(<100$ $\mathrm{nm}$ ) from the SC-GAS method than from the conventional method is evident in SEM pictures of the liposomes, where their occurrence was abundant. Smaller sized liposomes are delivered more efficiently to an infected site compared with larger sized liposomes. The SEM images in Fig. 4A and B confirm the occurrence of particles, supporting the micrographs from phase contrast microscopy. By observing the results regarding liposome size (Fig. 2G), and phase contrast and SEM images (Fig. 3K and $4 \mathrm{~A}$, respectively), the proposed configuration for SC-GAS liposome production, at 180 bar and $50{ }^{\circ} \mathrm{C}$ with $0.75 \%$ Tween 80 and a depressurization rate of $25 \mathrm{bar}^{\mathrm{min}^{-1}}$, can undoubtedly be employed to produce mono-dispersed nano-liposomes with reproducible features.

Liposome stability index (LSI). The liposome stability index (LSI) is a quick method to estimate the stability of liposomes. The process temperature, pressure and surfactant concentration had simultaneous effects on LSI. The liposomes were kept under stressed temperature conditions $\left(37 \pm 1^{\circ} \mathrm{C}\right)$ to trigger the process of aggregation. If the stability index value is lower, this is a sign of lesser stability in the liposomal formulation. As the pressure increases from 50 to 200 bar, the LSI is found to

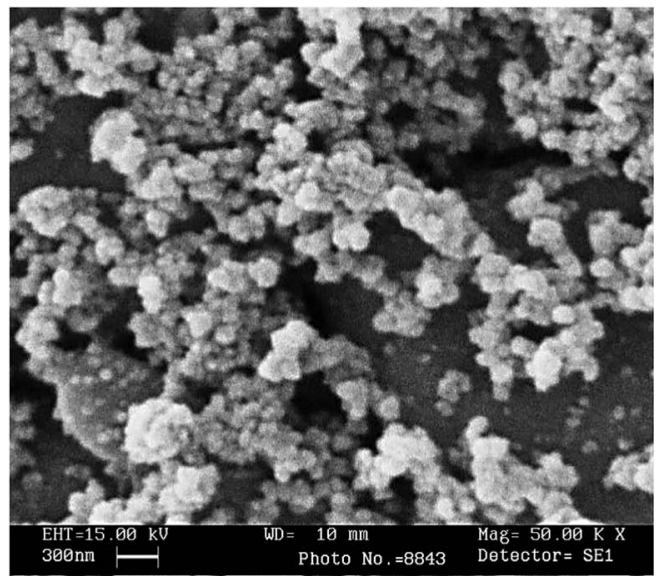

(A)

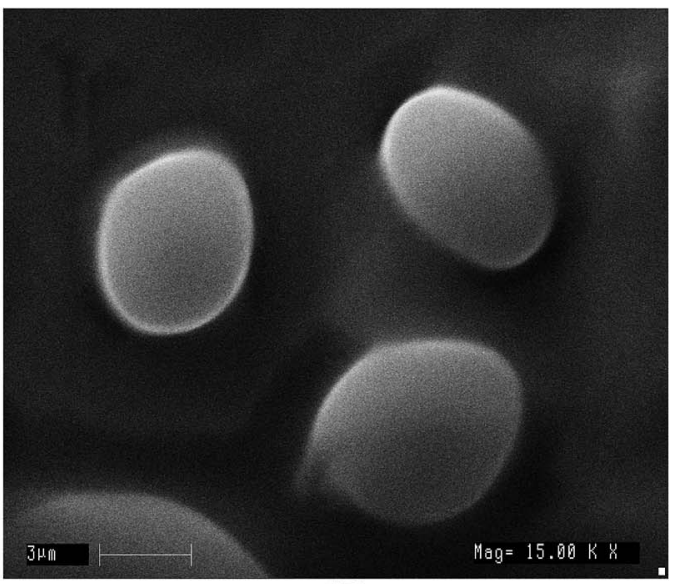

(B)

Fig. 4 SEM photomicrographs of liposomes prepared via: the $\mathrm{SCCO}_{2}$-GAS method at 180 bar, 50 and $0.75 \%$ Tween 80 (A); and the Bangham method (B). 
increase from 179 to 479 (Fig. S1 $\dagger$ ), indicating that a pressure between 170 and 200 bar is a favorable range to produce stabilized narrow size distributed nano-liposomes. When the temperature increases, the LSI increases (210-476) up to $50{ }^{\circ} \mathrm{C}$, but there was no significant difference when the temperature increased from 50 to $65^{\circ} \mathrm{C}$ (Fig. S1 $\dagger$ ). The emulsifying properties of the liposome system were improved when the Tween 80 concentration increased from 0 to $3 \%$, thus improving the LSI (129-698).

Viscosity of the liposomes. The viscosity of liposomes plays an important role during long term storage and is also an important parameter for stability and efficient drug release. Higher viscosity liposomes are an indication of smaller size and a narrow particle distribution. It is also known that higher viscosity nano-liposomes result in the slower release of active ingredients and have a lower clearance rate after administration. It was observed that all the liposome formulations showed near Newtonian behavior (Fig. S2 $\dagger$ ). The flow behavior of the liposomes was similar to milk, which is a good example of a stable $\mathrm{O} / \mathrm{W}$ emulsion where lipid droplets are stabilized by milk proteins. ${ }^{18,26}$ Table S1† explains the rheological properties of liposomes prepared under different formulation conditions. In the SC-GAS method, viscosity has a significant effect on particle size reduction and so the apparent viscosity of the liposome suspensions was found to increase with increasing pressure between 50 and 160 bar, and it was further stabilized between 160 and 200 bar. As the temperature increased the viscosity was found to increase and later it followed a slight decreasing trend. But an increase in the Tween 80 concentration from 0 to $3 \%$ increased the apparent viscosity of the liposomes from $1.16 \pm 0.01$ to $1.92 \pm 0.01 \mathrm{mPa}$ s. At a higher Tween 80 concentration, a larger amount of hydrophilic tails was available to entrap water molecules in the formulation, which may lead to increased viscosity. ${ }^{27}$ The highest apparent viscosity of $1.79 \pm 0.02 \mathrm{mPa}$ s was obtained for nano-liposomes prepared at 180 bar and $50{ }^{\circ} \mathrm{C}$, with $0.75 \%$ Tween 80 .

Comparison with the Bangham method. The physical properties of liposomes, in terms of mean particle size, EE, and $C_{\mathrm{u}}$, prepared via SC-GAS and thin-film methods were compared. The particle size distribution for liposomes prepared via the thin film method is shown in the Fig. $2 \mathrm{H}$. Liposome vesicles prepared via the conventional method have a relatively broad size distribution ranging between 0.55 and $10.05 \mu \mathrm{m}$ with a mean diameter of $5.64 \mu \mathrm{m}$, and moreover the vesicles exhibited irregular bilayer thickness (Fig. 3M \& 4B), which may reduce the membrane stability and lead to considerable leakage. The $C_{\mathrm{u}}$ and viscosity values were $11.97 \pm 0.019$ and $1.198 \pm 0.01$, which implies that the conventionally prepared liposomes were characterized as being highly poly-dispersed. The conventionally prepared liposomes showed poor EEs of $38.34 \pm 3.4,37.57 \pm 3.4$ and $33.45 \pm 3.3 \%$ for adenosine, cordycepin and polysaccharides, respectively. Another disadvantage of the conventional method is the poor reproducibility of its features. ${ }^{\mathbf{1 2}}$ The particle sizes of liposomes formed via the SCGAS method were markedly lower, exhibiting a thinner appearance (Fig. 5B) than those produced via a thin-film dispersion, which had higher heterogeneity and showed a very

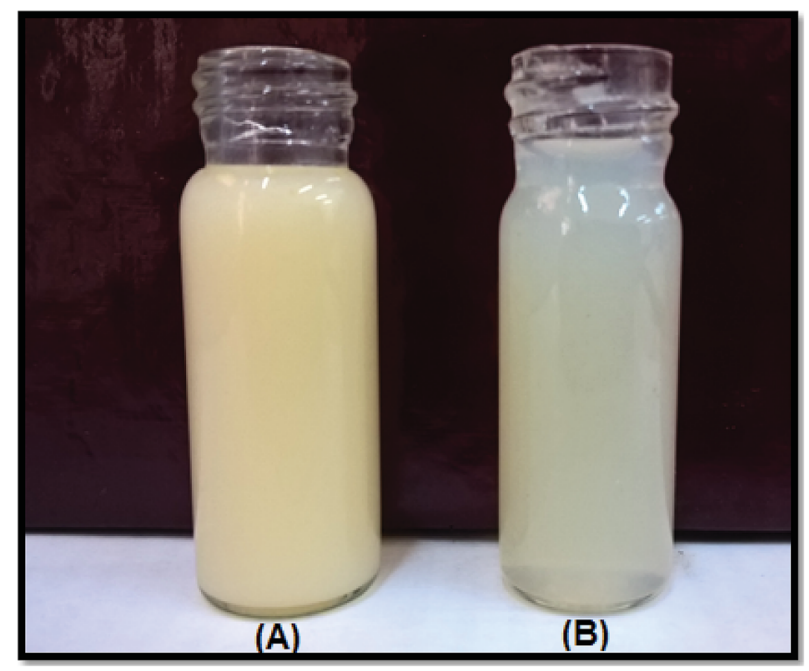

Fig. 5 CS liposomes prepared via the Bangham method (A); and the SC-GAS technique (B)

thick appearance (Fig. 5A). In comparison with the Bangham method, the SC-GAS process requires less energy, as discussed elsewhere, ${ }^{5}$ to yield nano-liposomes with a mean particle diameter of $0.072 \pm 0.002 \mu \mathrm{m}, C_{\mathrm{u}}$ of $1.10 \pm 0.012$ and an improved EE 1.97 times that achieved using the Bangham method (Fig. 3K).

The SC-GAS method in fed-batch mode is strongly supported as a superior method of nano-liposome production compared to other variants of SC techniques and high pressure based competitive methods that were discussed in an earlier report. ${ }^{5}$ The present configuration of the SC-GAS set-up facilitates nanoliposomes with a mean size of $72 \mathrm{~nm}$ and an $\mathrm{EE}$ of about $75 \%$, which is $40 \%$ less than other SC methods and 70-90\% less than high pressure homogenization and micro-fluidizer based techniques. This is the first study to report the production of stabilized superior liposomes that are less than $100 \mathrm{~nm}$ with a narrow range size distribution for the encapsulation of water soluble compounds from C. sinensis CS1197 via a SC-GAS method.

Thermal analysis of the nano-liposomes. The DSC method is a tool used to measure the temperature and energy variation involved in phase transitions, i.e., the gel to liquid-crystalline phase transitions of phospholipid bilayers, which reflects the degree of crystallinity of free $C$. sinensis CS1197 WE and extract entrapped in liposomes. DSC thermograms for free C. sinensis CS1197 and CS liposomes are shown in Fig. 6A. Empty liposomes (liposomes without extract) showed a melting endotherm at $137.94{ }^{\circ} \mathrm{C}$, CS nano-liposomes showed this at $139.50{ }^{\circ} \mathrm{C}$, and C. sinensis CS1197 WE does not show any melting endotherm. The melting endotherms are the result of crystallinity, and no peak was detected for free C. sinensis CS1197 WE; this may be due to the lack of crystalline forms and/or higher purity and an amorphous nature. The results were comparable to an earlier report. ${ }^{9}$ A liquid crystalline (LC) nature is very important for drug delivery, and LC based systems can offer the specific advantages of thermodynamic stability, high solubilization levels, improved bioavailability, protection against oxidation 


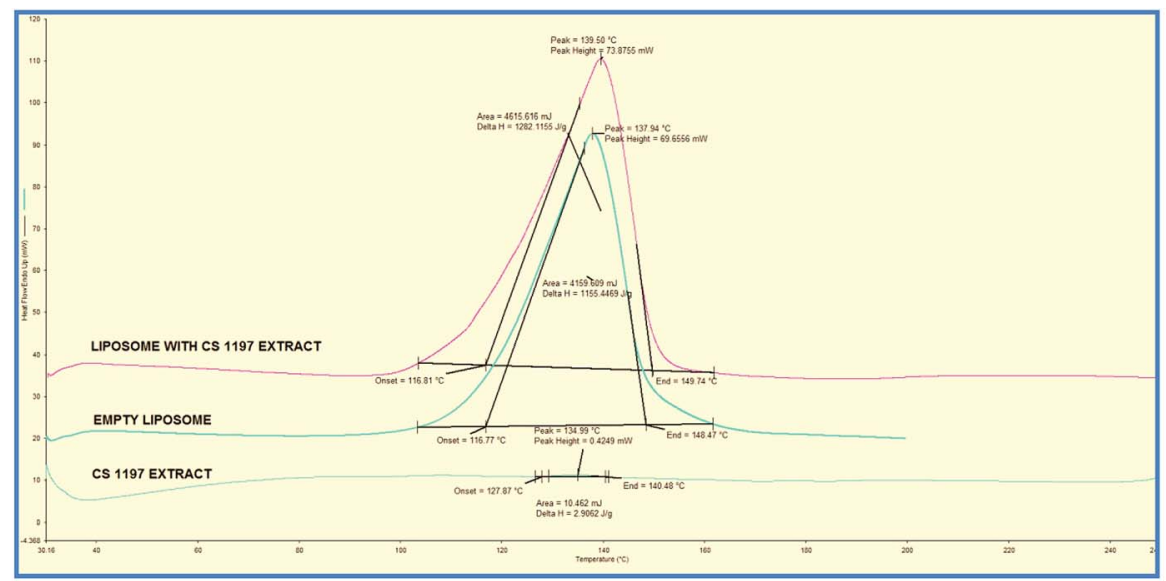

(A)

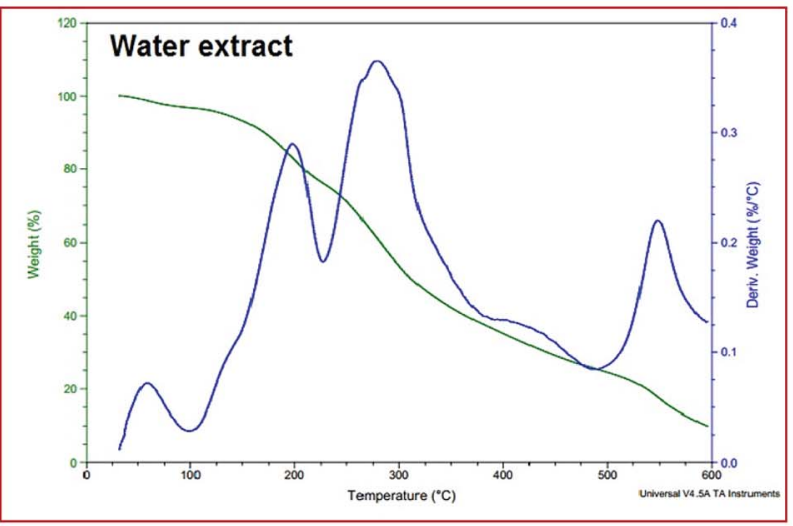

(B)

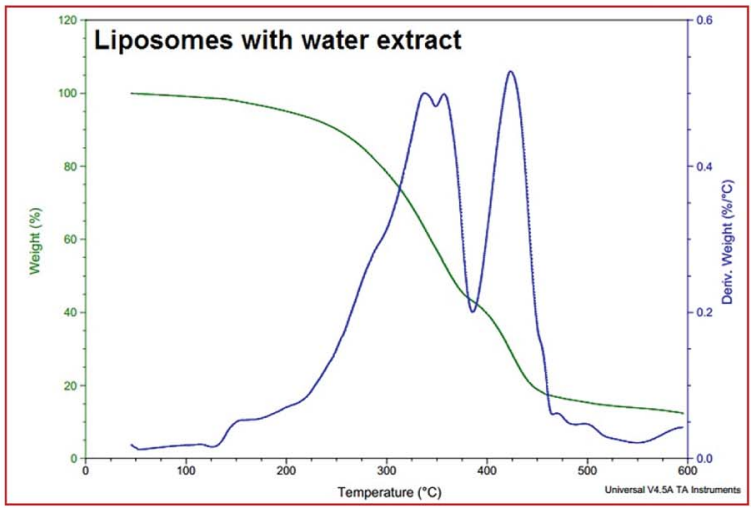

(C)

Fig. 6 DSC and TGA thermograms. DSC thermograms of CS1197 WE, empty liposomes $\left(T_{\circ}-116.77^{\circ} \mathrm{C}, T_{\mathrm{m}}-137.94^{\circ} \mathrm{C}, T_{\mathrm{C}}-148.47^{\circ} \mathrm{C}\right.$, and $\Delta H$ $\left.-1155 \mathrm{~J} \mathrm{~g}^{-1}\right)$ and $\mathrm{CS}$ nano-liposomes $\left(T_{\mathrm{o}}-116.81^{\circ} \mathrm{C}, T_{\mathrm{m}}-139.50{ }^{\circ} \mathrm{C}, T_{\mathrm{C}}-149.74{ }^{\circ} \mathrm{C}\right.$ and $\left.\Delta H-1282 \mathrm{~J} \mathrm{~g}^{-1}\right)(\mathrm{A})$; and TGA thermograms of $\mathrm{CS} 1197$ WE (B) and CS nano-liposomes (C).

and controlled release properties to pharmaceuticals. ${ }^{28}$ In the present study, crystallinity was attributed to free C. sinensis CS1197 WE due to liposomal encapsulation, which helps to enhance the performance of the formulation for drug delivery.

TGA was used to measure physical and chemical changes in compounds as a function of temperature. TGA curves for $C$. sinensis CS1197 WE and CS nano-liposomes are shown in Fig. 6B and C, respectively. During heating from 30 to $600{ }^{\circ} \mathrm{C}$, about $90 \%$ wt loss was observed at a temperature of $198^{\circ} \mathrm{C}$ for free C. sinensis CS1197 WE and $290{ }^{\circ} \mathrm{C}$ for the CS nano-liposomes. This wt loss may be due to a loss of moisture and/or a change in the molecular structure causing phase transitions (as shown by the secondary $Y$-axis). Liposomal encapsulation has improved the thermal stability of the extract.

FTIR analysis. FTIR spectra of $C$. sinensis CS1197 WE, empty liposomes and CS nano-liposomes were studied in order to understand the association between C. sinensis CS1197 water soluble compounds and phospholipids (Fig. 7). The FTIR spectrum of free $C$. sinensis CS1197 WE contains principle bands at 3402, 2931, 2360, 1627, 1404, 1080, and 1033 (Fig. 7A), and that of empty liposomes contains bands at 3410, 2924, 2854, 1735, 1651, 1465 and 1095 (Fig. 7B). The FTIR spectrum of CS liposomes (Fig. 7C) contains similar bands to those contained in both free extract and empty liposome spectra, indicating that the liposomal encapsulation of water soluble compounds did not result in the formation of new linkages, thus ensuring efficient drug unloading at the target sites.

XRD analysis. XRD has been widely used in the characterization of nanoparticles for detailing critical features, such as crystal structure, crystallite size, and strain. The broadening of diffraction peaks is due to disordered states of crystals. This has been attributed to the absence of total constructive and destructive interferences of X-rays in a finite sized lattice. ${ }^{29}$ The XRD pattern for CS nano-liposomes was recorded over a $2 \theta$ range of 0 to $80^{\circ}$, as shown in Fig. 8B. Sharp characteristic peaks appeared at $2 \theta=28^{\circ}$ and $41^{\circ}$, exhibiting a polymorphic nature. These diffraction peaks are due to an ordered lipid bi-layer, which is a superior characteristic of nano-liposomes produced via the SC-GAS method. However, no peak or broadened diffraction peak at $2 \theta=21^{\circ}$ was seen in the case of free $C$. sinensis CS1197 WE (Fig. 8A) which is due to its amorphous nature/lack of purity, or due to it being composed of highly disordered crystals. So, due to liposomal encapsulation, a highly ordered crystalline nature was attributed to C. sinensis CS1197 WE, which is important for enhanced performance in drug delivery. 
(A)

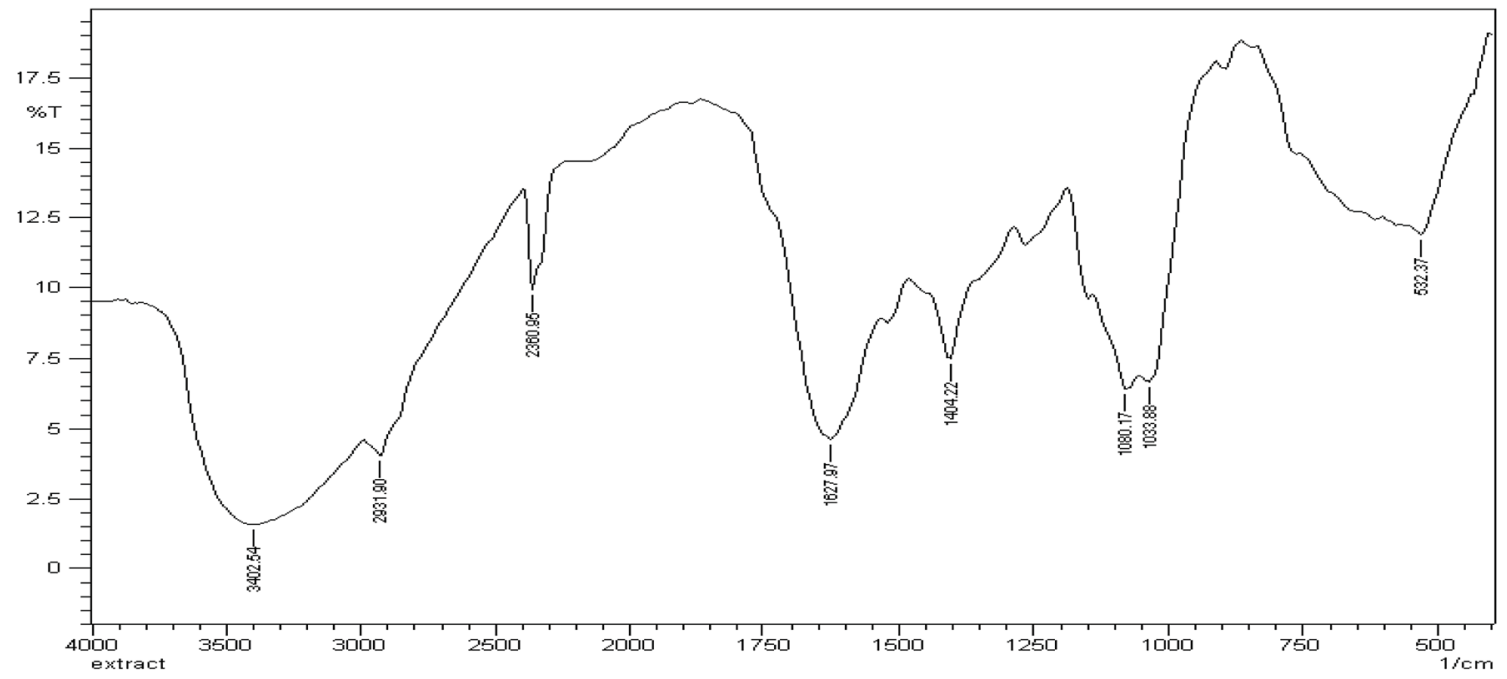

(B)

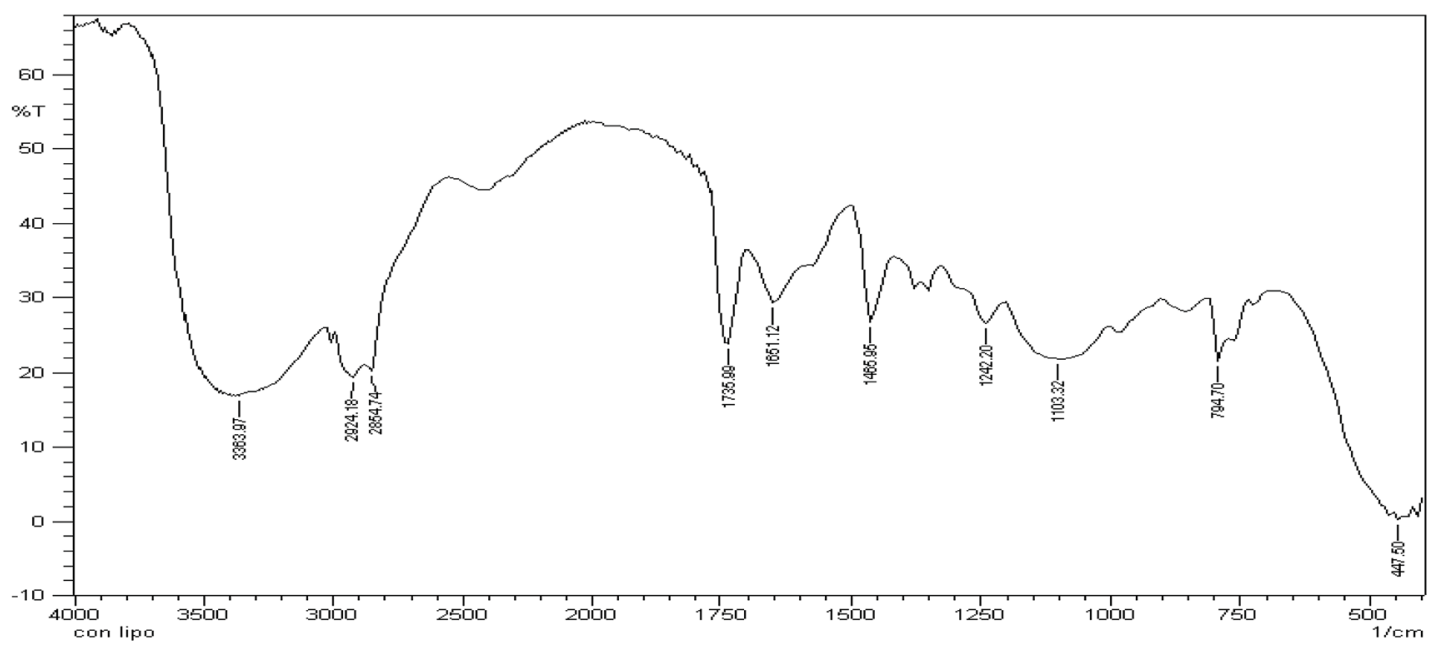

(C)

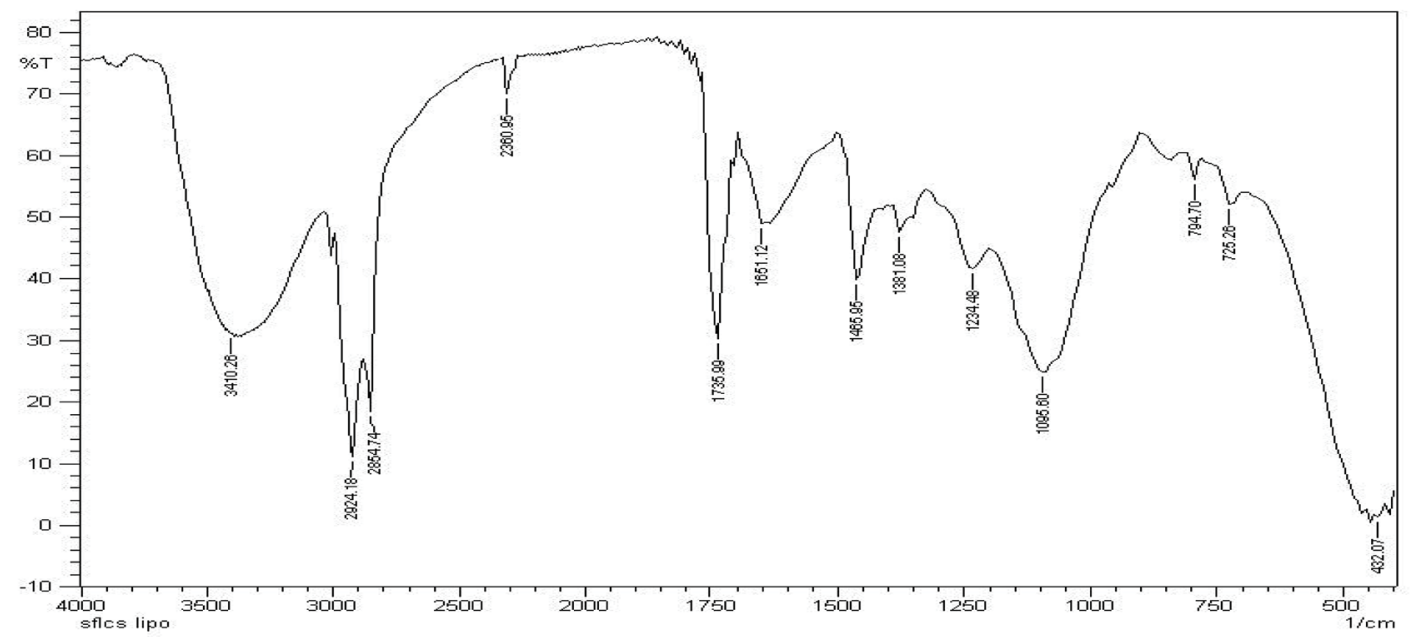

Fig. 7 FTIR spectra of: CS1197 water extract (A); empty liposomes (B); and CS liposomes (C) prepared via the SCCO 2 -GAS technique.

Storage studies. Storage stability is an important index of any drug delivery system. Physical instability can lead to drug leakage and the aggregation or fusion of vesicles. ${ }^{30}$ Liposomes prepared via the SC-GAS method were assessed after different time intervals of storage at 4,25 and $37^{\circ} \mathrm{C}$ and $\mathrm{pH}$ values of 2,4 , 7 and 10 (Table 1).
The nano-liposomes prepared via the SC-GAS method stored at 4,25 and $37^{\circ} \mathrm{C}$ were highly stable for up to 2 months (Table 1 ) with no significant changes $(p>0.05)$ in mean size, $C_{\mathrm{u}}$ and viscosity. This is due to the fact that uniformly distributed nanoliposomes possess a uniform charge density around the surface, attaining a thermodynamically stabilized state. Liposomes 


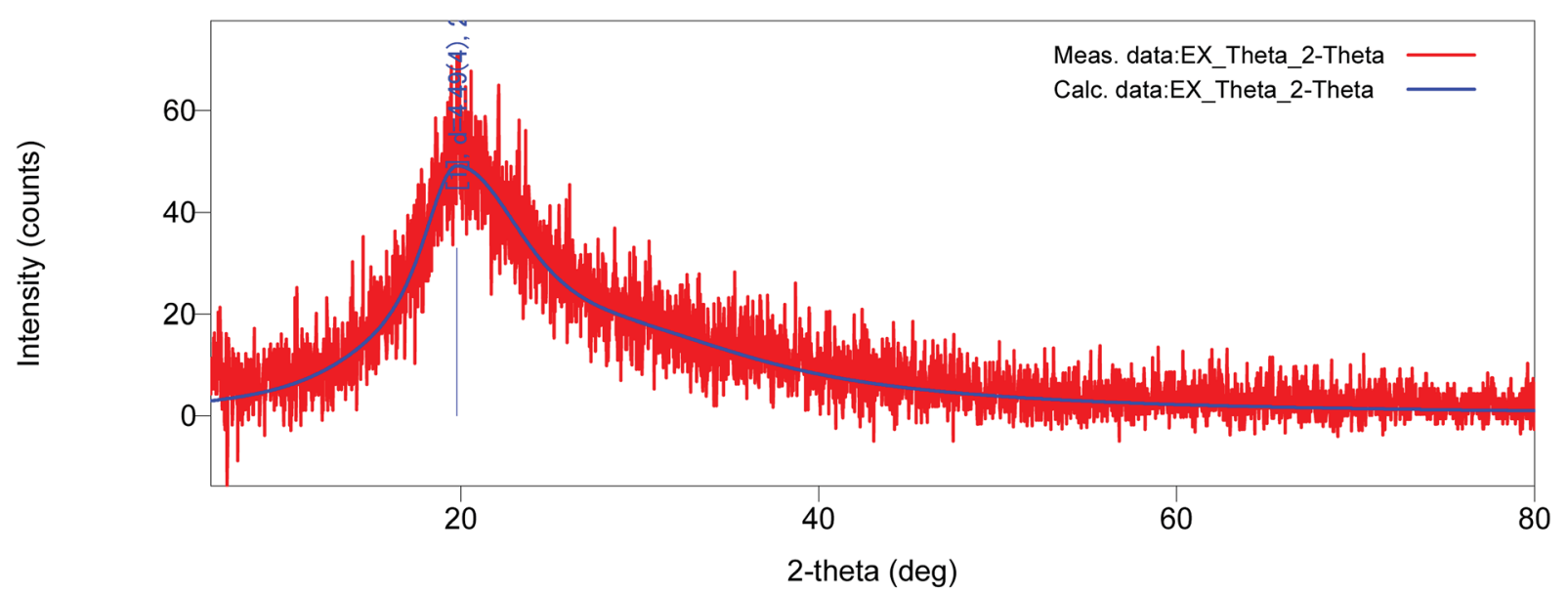

(A)

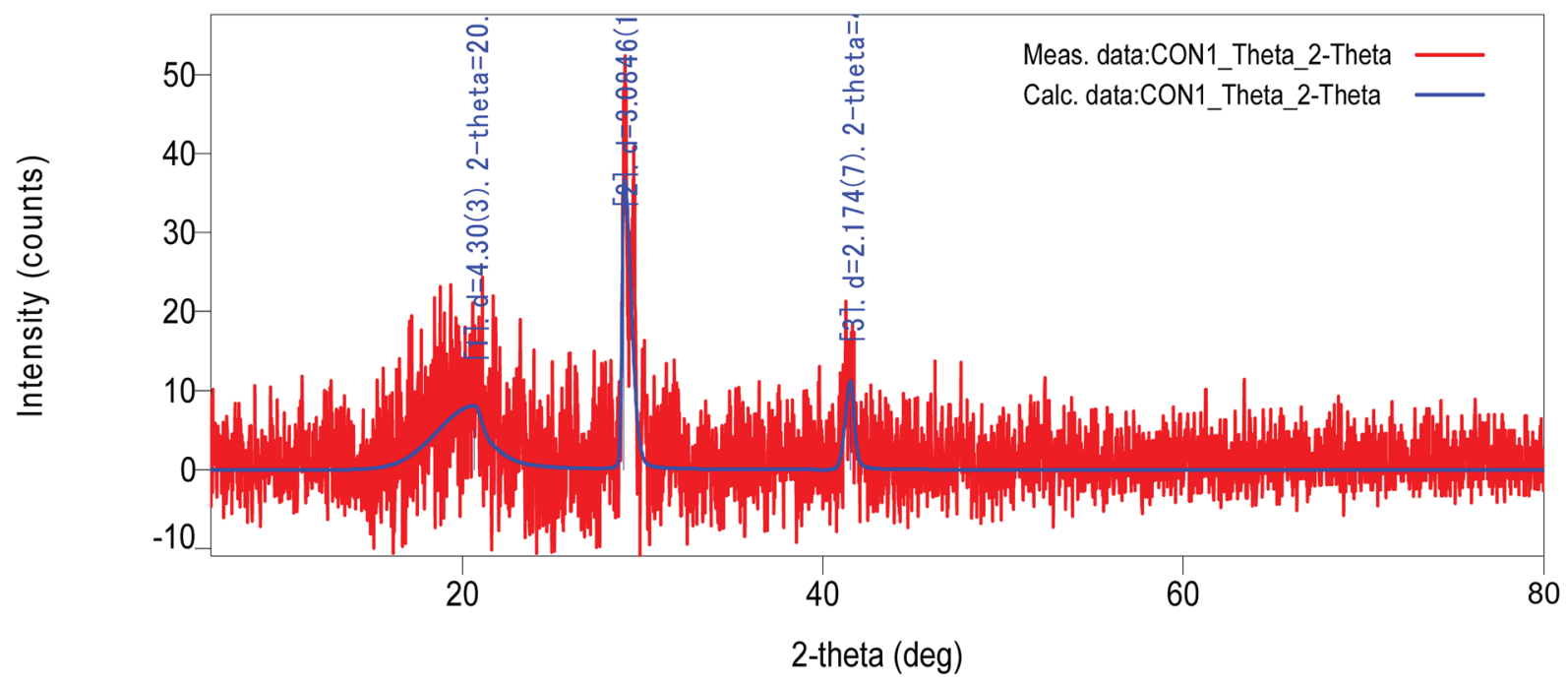

(B)

Fig. 8 X-ray diffraction spectra of: CS1197 WE (A); and CS nano-liposomes (B).

prepared via the SC-GAS and Bangham methods and stored at different temperatures were observed under a phase contrast microscope (Fig. 9). Liposomes prepared via the SC-GAS method showed no aggregation, but liposomes prepared via the Bangham method showed fast aggregation after 2 months. Large variations in the mean size caused an unequal distribution of the charge density i.e., large particles possessed a larger charge density compared to smaller particle, and as a result larger particles had the tendency to show dominance over smaller particles because the large potential difference caused aggregation. Fig. 10A-C shows the effects of storage temperature on the EE over two months. The EEs of liposomes for adenosine, cordycepin and polysaccharides decreased by less than $2.3 \%$ at $4{ }^{\circ} \mathrm{C}, 4.4 \%$ at $25{ }^{\circ} \mathrm{C}$ and $9.8 \%$ at $37{ }^{\circ} \mathrm{C}$ after one month, and less than $3.2 \%$ at $4{ }^{\circ} \mathrm{C}, 7.3 \%$ at $25{ }^{\circ} \mathrm{C}$ and $20.3 \%$ at $37{ }^{\circ} \mathrm{C}$ after two months. The study suggests that liposome storage at 4 and $25{ }^{\circ} \mathrm{C}$ favors minimal compound leakage, assuring $92-97 \%$ drug retention in the nano-liposomes, whereas $79-81 \%$ drug retention can be assured for liposomes stored at $37{ }^{\circ} \mathrm{C}$ (Table 2).
The CS nano-liposomes stored at different $\mathrm{pH}$ values of 2,4 , 7 and 10 were assessed for their EEs (Fig. 10D-F), mean diameters, $C_{\mathrm{u}}$ values and viscosities over two months (Table 1). The nano-liposomes stored at $\mathrm{pH} 4$ and 7 were stable for up to 2 months with no significant changes $(p>0.05)$ in mean size, $C_{\mathrm{u}}$ and viscosity. This is due to the fact that a $\mathrm{pH}$ value of 4 to 7 favored the maximum retention of surfactant molecules on the surfaces of the liposomes, thus arresting aggregation. However, nano-liposomes stored under extreme $\mathrm{pH}$ conditions, i.e., 2 and 10 , were stable until the $3^{\text {rd }}$ week and were later destabilized due to fast aggregation. Under extreme $\mathrm{pH}$ conditions, higher ionic strength caused a lowering of the surfactant charge density over the liposome surfaces, resulting in fast aggregation. This study suggests that liposome storage at $\mathrm{pH} 4$ and 7 favors minimal compound leakage, assuring $92-96 \%$ drug retention in nanoliposomes, whereas $68-80 \%$ drug retention can be assured for liposomes stored at $\mathrm{pH} 2$ and 10 (Table 2).

Oxidative stability of the CS nano-liposomes. Nanoliposomes prepared via the SC-GAS method were evaluated for 


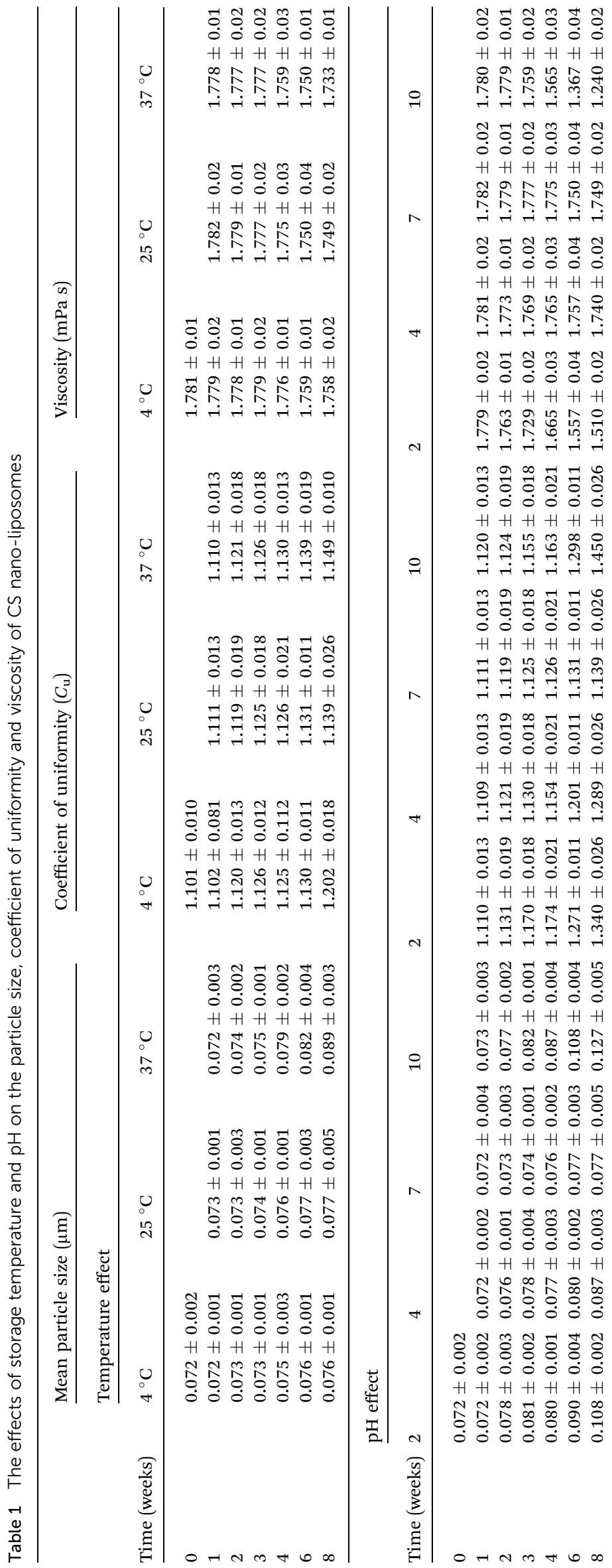




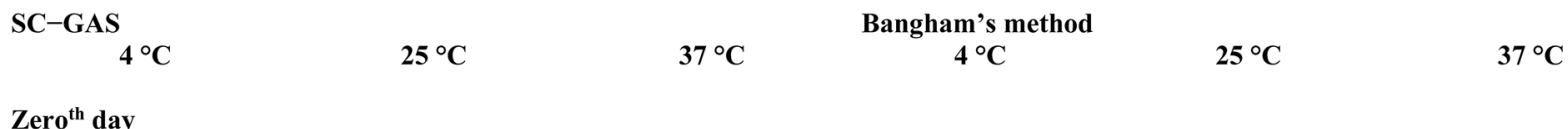
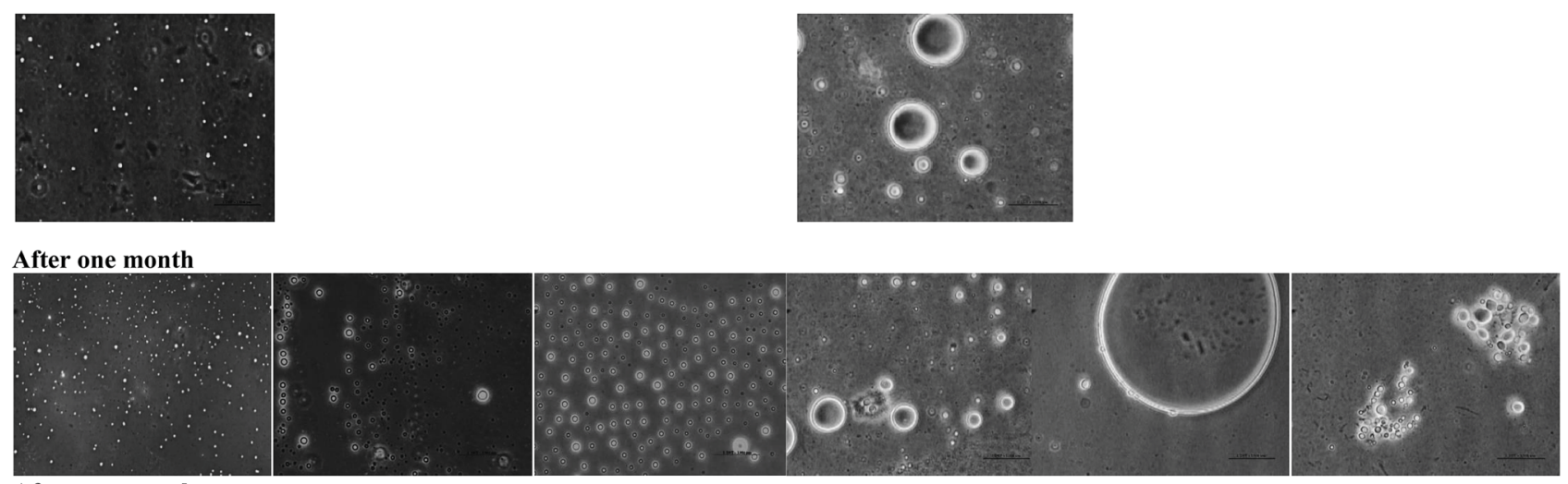

After two months

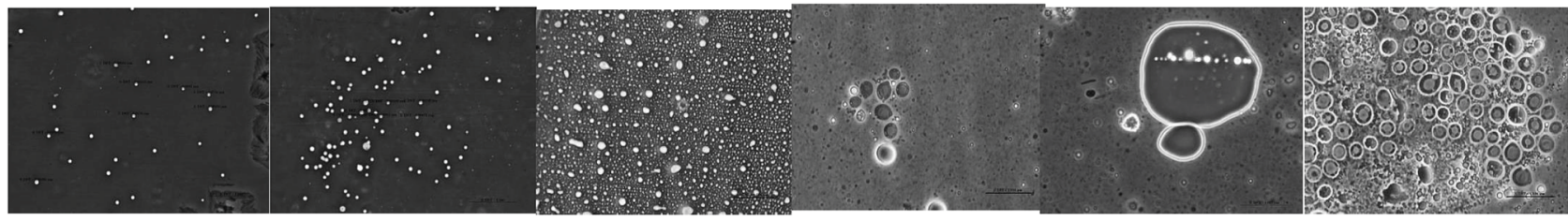

Fig. 9 Phase contrast micrographs of liposomes prepared via the SC-GAS and Bangham methods, stored at different temperatures.

their oxidative stability at different temperatures and $\mathrm{pH}$ values over 8 weeks (Fig. 11). Liposomes stored at 4 and $25^{\circ} \mathrm{C}$ showed increasing PVs for the first 4 weeks and were later found to stabilize. The maximum PVs for liposomes at 4 and $25{ }^{\circ} \mathrm{C}$ were found to be 1.15 and 1.53 meq $\mathrm{O}_{2} / \mathrm{kg}$ of sample after 8 weeks. However, liposomes stored at $37{ }^{\circ} \mathrm{C}$ exhibited the highest PV, i.e., 4.88 meq $\mathrm{O}_{2} / \mathrm{kg}$ of sample. It can be concluded that liposome storage at 4 and $25{ }^{\circ} \mathrm{C}$ can ensure minimum lipid oxidation. In addition, the oxidative status of nano-liposomes at different $\mathrm{pH}$ values was evaluated over 8 weeks (Fig. 11). The liposomes stored at $\mathrm{pH} 4$ and 7 showed minimal PVs, i.e., 0.32, 1.30 , and 1.58 meq $\mathrm{O}_{2} \mathrm{~kg}$ of sample. Storage at $\mathrm{pH} 2$ showed the minimum PV, but the storage of liposomes at $\mathrm{pH} 2$ cannot be recommended because of the increased particle size and decreased $\mathrm{EE}$ at a low $\mathrm{pH}$ value of 2. Liposome storage at a higher $\mathrm{pH}$ value of 10 resulted in accelerated lipid oxidation, and the highest PV was 2.02 meq $\mathrm{O}_{2} / \mathrm{kg}$ of sample. So from the results, it can be concluded that extreme $\mathrm{pH}$ conditions are not recommended, and $\mathrm{pH}$ values of 4 and 7 favor minimal lipid oxidation.
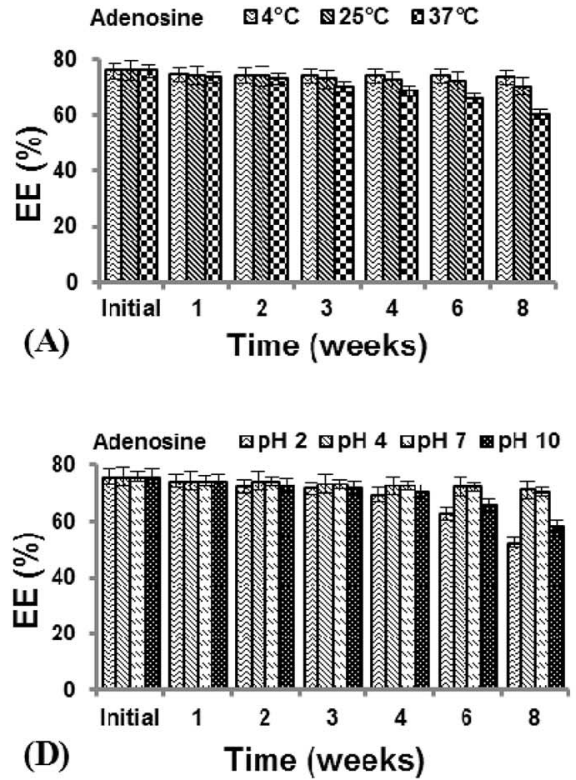
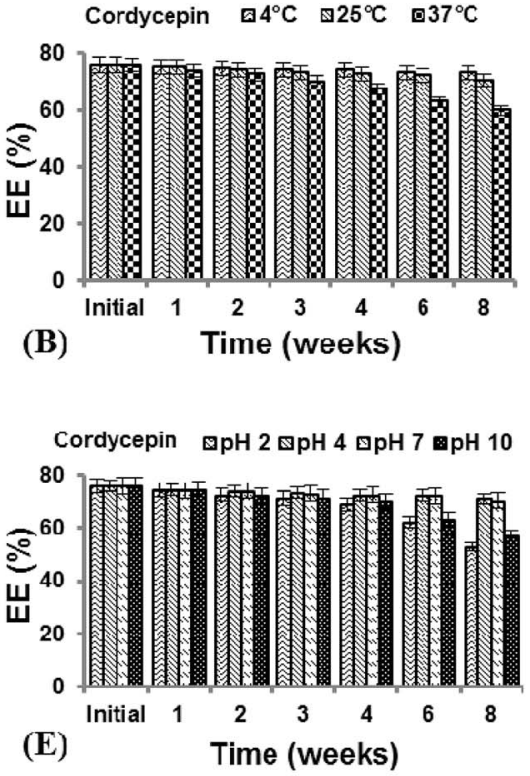
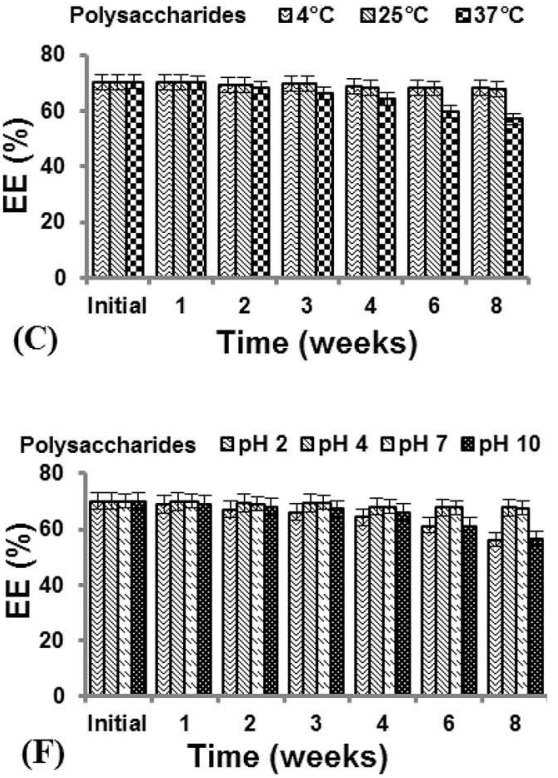

Fig. 10 The effects of storage time, temperature and pH on the encapsulation efficiencies of CS nano-liposomes. 
Table 2 The effects of storage temperature and $\mathrm{pH}$ on the percentage retention of water soluble compounds from CS1197 in the nanoliposomes

\begin{tabular}{lllll}
\hline \multirow{2}{*}{ Parameter } & \multicolumn{4}{l}{ Retention after 8 weeks (\%) } \\
\cline { 3 - 5 } & & Adenosine & Cordycepin & Polysaccharides \\
\hline \multirow{2}{*}{ Temperature $\left({ }^{\circ} \mathrm{C}\right)$} & 4 & 97.07 & 96.26 & 96.79 \\
& 25 & 92.65 & 92.83 & 96.51 \\
$\mathrm{pH}$ & 37 & 79.6 & 78.82 & 81.17 \\
& 2 & 68.79 & 69.42 & 79.9 \\
& 4 & 93.64 & 93.44 & 96.66 \\
& 7 & 92.65 & 92.83 & 96.51 \\
& 10 & 76.96 & 75.07 & 80.69 \\
\hline
\end{tabular}

In vitro release studies. In vitro drug release studies are important to help understand the in vivo performance of the dosage form. Drug release studies help in the evaluation of sustained and prolonged release dispersion systems. ${ }^{31}$ In the present study, in vitro release studies of adenosine, cordycepin and polysaccharides were carried over $48 \mathrm{~h}$ through a dialysis membrane. Fig. 12 shows that $C$. sinensis CS1197 WE in its free form resulted in a fast release profile, i.e., release values of about 81,83 , and $51 \%$ for adenosine, cordycepin, and polysaccharides, respectively, were observed over $4 \mathrm{~h}$. But in the case of the nano-liposomes, release values of about 40, 46 and $27 \%$ for adenosine, cordycepin and polysaccharides, respectively (Fig. 12) were observed over $4 \mathrm{~h}$. It is suggested that the drug release from the liposomes characteristically exhibited a biphasic fashion, i.e., an initial fast drug loss followed by diffusion through the membrane. ${ }^{32}$ The initial fast rate of release is commonly due to compound detachment from the liposomal surface, while the later slow release results from sustained drug release from the inner lamellae. C. sinensis CS1197 WE is mainly entrapped inside the bilayer lipid structure of the liposomes and the in vitro release studies showed that compound transport out of the liposomes was driven mainly by a diffusion-
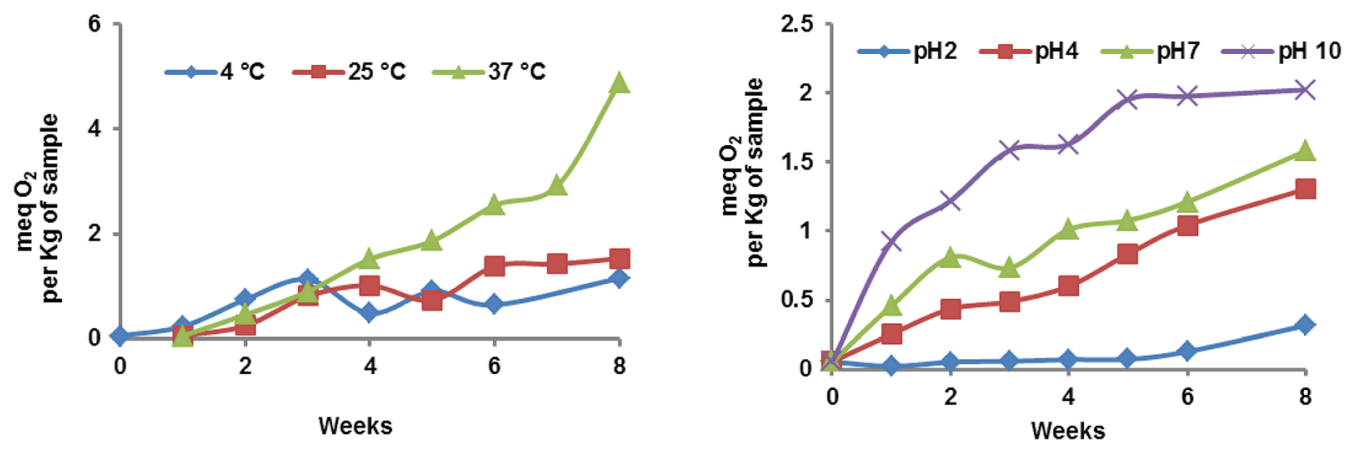

Fig. 11 Peroxide values (PVs) for CS nano-liposomes observed for 8 weeks under accelerated conditions of temperature and pH.

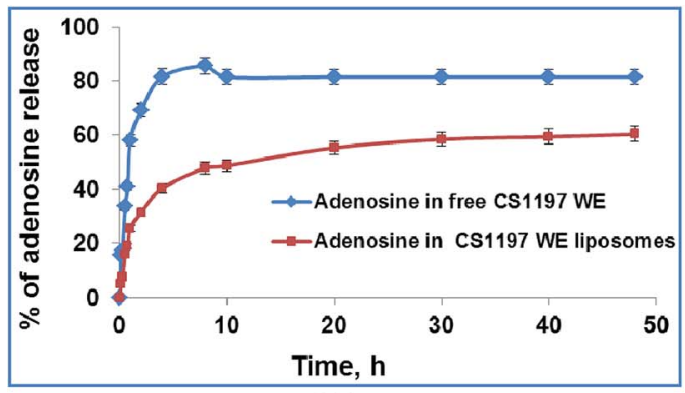

(A)

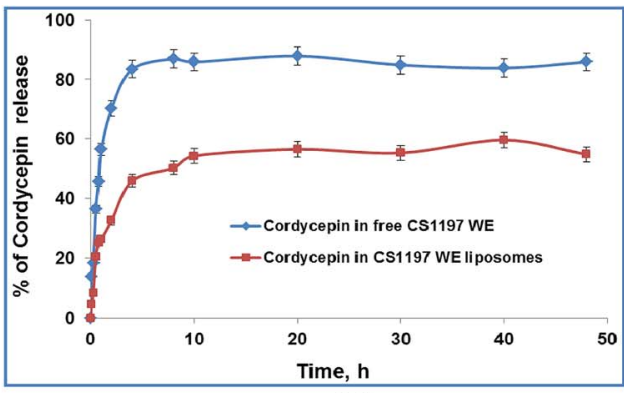

(B)

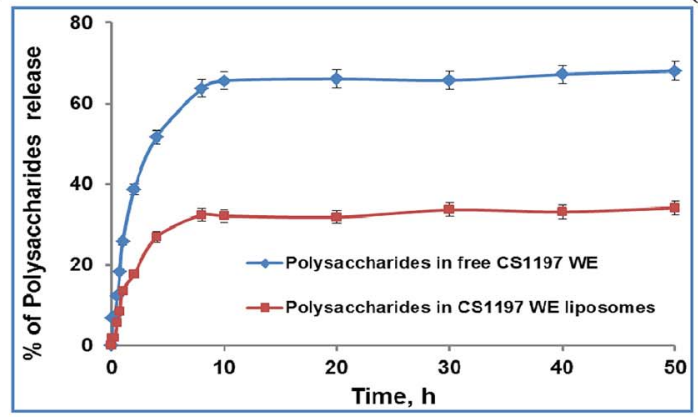

(C)

Fig. 12 The in vitro kinetic release profiles of CS nano-liposome dispersions prepared via the SC-GAS method in pH 6.8 hydration medium at $37^{\circ} \mathrm{C}$. 
Table 3 The parameters of the kinetic models for in vitro release by the CS nano-liposome formulations

\begin{tabular}{lllllll}
\hline & & \multicolumn{2}{l}{ First order } & & \multicolumn{2}{l}{ Higuchi model } \\
& & $K_{1}$ & $R^{2}$ & & $K_{2}$ & $R^{2}$ \\
\hline \multirow{2}{*}{ Free CS1197 WE } & Adenosine & 0.1794 & 0.927 & & 26.622 & 0.833 \\
& Cordycepin & 0.191 & 0.943 & & 27.594 & 0.847 \\
& Polysaccharides & 0.017 & 0.929 & & 22.512 & 0.969 \\
& Adenosine & 0.027 & 0.849 & & 17.877 & 0.953 \\
& Cordycepin & 0.031 & 0.844 & & 17.274 & 0.931 \\
& Polysaccharides & 0.047 & 0.869 & & 11.593 & 0.957
\end{tabular}

controlled mechanism. Since prolonged delivery through nanoliposomes prepared via the SC-GAS method undoubtedly reflects a longer residence time in circulation, this could help bio-active compounds to reach target sites.

The mechanism of the release phenomenon through the nano-liposomes was studied via applying two kinetic models. The kinetic data for the two models is shown in Table 3 and the regression of the kinetic models to the in vitro kinetic release data from the CS nano-liposome formulations is shown in Fig. S3. $\dagger$ A first order model fits the in vitro experimental release data of free C. sinensis CS1197 WE well $\left(R^{2}=0.927-0.943\right)$, where a fast release pattern is adopted. But the release of $C$. sinensis CS1197 WE through the nano-liposomes followed the Higuchi model $\left(R^{2}=0.931-0.957\right)$, proving that release through the liposomes involves a diffusion-controlled mechanism.

\section{Conclusions}

The SC-GAS method provides a simpler way to prepare liposomes with a characteristic nano-size and better morphology (i.e., a uniform size and shape). The current configuration of the SC-GAS mediated liposome production unit can be operated in fed-batch mode, with the additional feature of ensuring reproducibility for the encapsulation of both hydrophilic and hydrophobic active compounds. CS nano-liposome production under optimized conditions, i.e., $180 \mathrm{bar}, 50{ }^{\circ} \mathrm{C}$ and $0.75 \%$ Tween 80, offers stabilized liposomes exhibiting good LSI and rheological properties and achieving about $75 \%$ EE. DSC and TGA studies reveal that encapsulation within a lipid bilayer improves the thermal stability of $C$. sinensis water soluble compounds. FTIR analysis proved that the liposomal encapsulation of $C$. sinensis WE ensures the efficient unloading of active ingredients at target sites. The ordered structure of the lipid bilayer was attributed to crystallinity, which was confirmed via XRD analysis. Storage and oxidative stability studies suggest that maintaining the CS nano-liposomes at 4 and $25{ }^{\circ} \mathrm{C}$ ensures maximum drug retention and minimum lipid oxidation. In vitro kinetic release studies show that the active compounds are delivered through liposomes via a diffusion controlled mechanism. The proposed model can be successfully implemented on a pilot scale, indicating that supercritical processes can provide promising technical and economic conditions for large scale production. Supercritical technology shows advantages over conventional preparation methods and offers great potential for the encapsulation of valuable compounds relevant to nutraceutical, functional food and pharmaceutical applications.

\section{Conflicts of interest}

The authors report no conflicts of interest. The authors alone are responsible for the content and writing of this paper.

\section{Acknowledgements}

The author (SMG) acknowledges encouragement from the Director of the CSIR - Central Food Technological Research Institute, Mysore, India.

\section{References}

1 P. LI, H. JI, T. Tina and W. Karl, Chin. J. Pharm. Anal., 2001, 21, 77-78.

2 M. Shashidhar, P. Giridhar, K. U. Sankar and B. Manohar, J. Funct. Foods, 2013, 5, 1013-1030.

3 G. Gregoriadis, N. Engl. J. Med., 1976, 295, 704-710.

4 S.-L. Huang, Adv. Drug Delivery Rev., 2008, 60, 1167-1176.

5 G. Shashidhar and P. Gadkari, RSC Adv., 2016, 6, 5773957750.

6 S. Varona, Á. Martín and M. a. J. Cocero, Ind. Eng. Chem. Res., 2011, 50, 2088-2097.

7 A. S. Zarena and K. U. Sankar, Ther. Delivery, 2011, 2, 259277.

8 U. S. Kadimi, D. R. Balasubramanian, U. R. Ganni, M. Balaraman and V. Govindarajulu, Nanomedicine, 2007, 3, 273-280.

9 S. M. Ghatnur, R. S. Sonale, M. Balaraman and U. S. Kadimi, J. Liposome Res., 2012, 22, 215-223.

10 L. Zhao and F. Temelli, J. Supercrit. Fluids, 2015, 100, 110120.

11 L. Zhao and F. Temelli, J. Food Eng., 2015, 158, 104-112.

12 L. Zhao, F. Temelli, J. M. Curtis and L. Chen, Food Res. Int., 2015, 77, 63-72.

13 I. E. Santo, R. Campardelli, E. C. Albuquerque, S. V. de Melo, G. Della Porta and E. Reverchon, Chem. Eng. J., 2014, 249, 153-159.

14 S. M. Ghatnur, G. Parvatam and M. Balaraman, Pharmacogn. Mag., 2015, 11, 448.

15 A. Bangham, M. M. Standish and J. Watkins, J. Mol. Biol., 1965, 13, 238-IN227.

16 R. F. Craig, Craig's soil mechanics, CRC Press, 2004.

17 K. N. Pearce and J. E. Kinsella, J. Agric. Food Chem., 1978, 26, 716-723.

18 P. V. Gadkari and M. Balaraman, J. Food Eng., 2015, 147, 1423.

19 R. Wrolstad, T. Acree, E. Decker, M. Penner, D. Reid, S. Schwartz, C. Shoemaker, D. Smith and P. Sporns, Lipids, and Carbohydrates. Wiley, New Jersey, 2005.

20 T. P. Castor and L. Chu, US Pat., US5776486A, 1998.

21 T. Castor, WO9427581, 1994.

22 R. Campardelli and E. Reverchon, J. Food Eng., 2015, 149, 131-136. 
23 G. D. Bothun, B. L. Knutson, H. J. Strobel and S. E. Nokes, Langmuir, 2005, 21, 530-536.

24 L. Lesoin, O. Boutin, C. Crampon and E. Badens, Colloids Surf., A, 2011, 377, 1-14.

25 C. T. Lee, P. A. Psathas, K. P. Johnston, J. deGrazia and T. W. Randolph, Langmuir, 1999, 15, 6781-6791.

26 A. Zarena, S. Bhattacharya and U. S. Kadimi, Food Bioprocess Technol., 2012, 5, 3007-3013.

27 J. Rao and D. J. McClements, J. Agric. Food Chem., 2011, 59, 5026-5035.
28 P. P. Gaikwad and T. Desai Maya, Int. J. Pharma Res. Rev., 2013, 2, 40-52.

29 B. Akbari, M. P. Tavandashti and M. Zandrahimi, Iran. J. Mater. Sci. Eng., 2011, 8, 48-56.

30 W. Zhou, W. Liu, L. Zou, W. Liu, C. Liu, R. Liang and J. Chen, Colloids Surf., B, 2014, 117, 330-337.

31 V. Venkateswarlu and K. Manjunath, J. Controlled Release, 2004, 95, 627-638.

32 P. Panwar, B. Pandey, P. Lakhera and K. Singh, Int. J. Nanomed., 2010, 5, 101-108. 